\title{
Second order mean field games with degenerate diffusion and local coupling
}

\author{
Pierre Cardaliaguet, P. Jameson Graber, Alessio Porretta and \\ Daniela Tonon
}

\begin{abstract}
We analyze a (possibly degenerate) second order mean field games system of partial differential equations. The distinguishing features of the model considered are (1) that it is not uniformly parabolic, including the first order case as a possibility, and (2) the coupling is a local operator on the density. As a result we look for weak, not smooth, solutions. Our main result is the existence and uniqueness of suitably defined weak solutions, which are characterized as minimizers of two optimal control problems. We also show that such solutions are stable with respect to the data, so that in particular the degenerate case can be approximated by a uniformly parabolic (viscous) perturbation.
\end{abstract}

Mathematics Subject Classification. 35K55, 49N70.

\section{Introduction}

This paper is devoted to the analysis of second order mean field games systems with a local coupling. The general form of these systems is:

$$
\left\{\begin{array}{l}
\left(\text { i) }-\partial_{t} \phi-A_{i j} \partial_{i j} \phi+H(x, D \phi)=f(x, m(t, x))\right. \\
\text { (ii) } \partial_{t} m-\partial_{i j}\left(A_{i j} m\right)-\operatorname{div}\left(m D_{p} H(x, D \phi)\right)=0 \\
\text { (iii) } m(0)=m_{0}, \phi(x, T)=\phi_{T}(x)
\end{array}\right.
$$

where $A: \mathbb{R}^{d} \rightarrow \mathbb{R}^{d \times d}$ is symmetric and nonnegative, the Hamiltonian $H$ : $\mathbb{R}^{d} \times \mathbb{R}^{d} \rightarrow \mathbb{R}$ is convex in the second variable, the coupling $f: \mathbb{R}^{d} \times[0,+\infty) \rightarrow$ $[0,+\infty)$ is increasing with respect to the second variable, $m_{0}$ is a probability density and $\phi_{T}: \mathbb{R}^{d} \rightarrow \mathbb{R}$ is a given function. The functions $H$ and $f$, and the matrix $A$, could as well depend on time, but since this does not give any additional difficulty, we will avoid it just to simplify notations.

Mean field game systems (MFG systems) have been introduced simultaneously by Lasry and Lions [17-19,21] and Huang et al. [15] to describe Nash equilibria in differential games with infinitely many players. The first unknown 
$\phi=\phi(t, x)$ is the value function of an optimal control problem of a typical small player. In this control problem, the dynamics is given by the controlled stochastic differential equation

$$
d X_{s}=v_{s} d s+\Sigma\left(X_{s}\right) d B_{s}
$$

where $\left(v_{s}\right)$ is the control, $\left(B_{s}\right)$ is a Brownian motion and $\Sigma \Sigma^{T}=A$. The cost is given by

$$
\mathbb{E}\left[\int_{0}^{T} H^{*}\left(X_{s},-v_{s}\right)+f\left(X_{s}, m\left(s, X_{s}\right)\right) d s+\phi_{T}\left(X_{T}\right)\right]
$$

where $H^{*}$ is the Fenchel conjugate of $H$ with respect to the second variable. For each time $t \in[0, T]$ the quantity $m(t, x)$ denotes the density of population of small players at position $x$. In the control problem the term involving $f$ formalizes the fact that the cost of the player depends on this density $m$. As $\phi$ is the value function of this control problem, the optimal control of a typical small player is formally given by the feedback $(t, x) \rightarrow-D_{p} H(x, D \phi(t, x))$. Hence the second equation (1)-(ii) is the Kolmogorov equation of the process $\left(X_{s}\right)$ when the small player plays in an optimal way. By the mean field approach, this equation also describes the evolution of the whole population density as all players play in an optimal way.

MFG systems with uniformly parabolic diffusions - typically $A_{i j} \partial_{i j} \phi=$ $\Delta \phi$-have been the object of several contributions, either by PDE methods (see, e.g., [7,12,13,17-19,21,23]) or by stochastic techniques (see, e.g., [2,15]): in this setting one often expects the solutions to be smooth, at least if the coupling is nonlocal and regularizing or if it has a "small growth". This is still the case for a logarithmic coupling: $f(m)=\ln (m)$, as recently proved in [11]. The case of local couplings with an arbitrary growth has been discussed in [7] for purely quadratic hamiltonians (i.e. $H=|D \phi|^{2}$ ), in which case solutions are proved to be smooth, and in [23] for general hamiltonians, by proving existence and uniqueness of weak solutions. Here we concentrate on degenerate parabolic equations. In this case the usual fixed point techniques used to prove the existence of solutions in the uniformly parabolic setting break down by lack of regularity. One then has to rely on convex optimization methods: this idea, which goes back to the analysis of some optimal transport problems (see $[1,5]$ ), has already been used to study first order MFG systems (i.e., $A \equiv 0$ ): see $[3,4,14]$. However it was not clear in these papers wether the weak solution was stable with respect to viscous approximation, i.e., if we could obtain weak solutions of the first order MFG systems by passing to the limit in uniformly parabolic ones. This issue has partially motivated our study.

In this paper we show the existence and uniqueness of a weak solution for the degenerate mean field game system (1) as well as the stability of solutions with respect to perturbation of the data: this includes of course stability by viscous approximation.

Concerning existence and uniqueness of solutions, the paper improves the existing results in two directions. First we consider non uniformly parabolic 
second order MFG systems, which have never been considered before. The introduction of second order derivatives induces several issues: in particular, in contrast with the first order equations, we do not expect the function $\phi$ to be BV (as in $[4,14]$ ), which obliges us to be very careful about trace properties. Secondly - and this is new even for first order MFG systems - we drop a restriction between the growth condition of $H$ and the growth condition of $f$, restriction which was mandatory in the previous papers: see [3,4]. To overcome the difficulty, we provide new integral estimates for subsolutions of HamiltonJacobi equations with unbounded right-hand side (Theorems 3.1 and 3.3). We think that these results are of independent interest.

With these estimates in hand, the structure of proof for the existence and uniqueness follows roughly the lines already developed in $[3-5,14]$ : basically it amounts to show that the MFG system can be viewed as an optimality condition for two convex problems, the first one being an optimal control of Hamilton-Jacobi equation, the second one an optimal control problem for the Fokker-Planck equation (see Sect. 4 for details). A byproduct of this approach is the stability of weak solutions with respect to the data (Theorem 6.5), which can be obtained by $\Gamma$-convergence techniques.

The paper is organized as follows. First we introduce the notation and assumptions needed throughout the paper (Sect. 2). Then (Sect. 3) we give our new estimates for subsolutions of Hamilton-Jacobi equations with a superlinear growth in the gradient variable and an unbounded right-hand side. In Sect. 4, we introduce the two optimal control problems and show that they are in duality while in Sect. 5 we show that the optimal control problem for the Hamilton-Jacobi equation has a "relaxed solution." Section 6 is devoted to the analysis of the MFG system (existence, uniqueness and characterization). In the last section we discuss the stability of solutions.

\section{Notations and assumptions}

Notations We denote by $\langle x, y\rangle$ the Euclidean scalar product of two vectors $x, y \in \mathbb{R}^{d}$ and by $|x|$ the Euclidean norm of $x$. We use conventions on repeated indices: for instance, if $a, b \in \mathbb{R}^{d}$, we often write $a_{i} b_{i}$ for the scalar product $\langle a, b\rangle$. More generally, if $A$ and $B$ are two square symmetric matrices of size $d \times d$, we write $A_{i j} B_{i j}$ for $\operatorname{Tr}(A B)$.

To avoid further difficulties arising from boundary issues, we work in the flat $d$-dimensional torus $\mathbb{T}^{d}=\mathbb{R}^{d} \backslash \mathbb{Z}^{d}$. We denote by $P\left(\mathbb{T}^{d}\right)$ the set of Borel probability measures over $\mathbb{T}^{d}$. It is endowed with the Kantorovich-Rubinstein distance (which metricizes the weak-* convergence):

$$
\mathbf{d}_{\mathbf{1}}\left(m, m^{\prime}\right):=\sup _{\phi} \int_{\mathbb{T}^{d}} \phi d\left(m-m^{\prime}\right)
$$

where the supremum is taken over the set of Lipschitz continuous maps $\phi$ : $\mathbb{T}^{d} \rightarrow \mathbb{R}$ which are Lipschitz continuous of constant 1 .

For $k, n \in \mathbb{N}$ and $T>0$, we denote by $\mathcal{C}^{k}\left([0, T] \times \mathbb{T}^{d}, \mathbb{R}^{n}\right)$ the space of maps $\phi=\phi(t, x)$ of class $\mathcal{C}^{k}$ in time and space with values in $\mathbb{R}^{n}$. For $p \in[1, \infty]$ 
and $T>0$, we denote by $L^{p}\left(\mathbb{T}^{d}\right)$ and $L^{p}\left((0, T) \times \mathbb{T}^{d}\right)$ the set of $p$-integrable maps over $\mathbb{T}^{d}$ and $[0, T] \times \mathbb{T}^{d}$ respectively. We often abbreviate $L^{p}\left(\mathbb{T}^{d}\right)$ and $L^{p}\left((0, T) \times \mathbb{T}^{d}\right)$ into $L^{p}$. We denote by $\|f\|_{p}$ the $L^{p}$-norm of a map $f \in L^{p}$. Assumptions We now collect the assumptions on the coupling $f$, the Hamiltonian $H$ and the initial and terminal conditions $m_{0}$ and $\phi_{T}$. These conditions are supposed to hold throughout the paper.

(H1) (Conditions on the coupling) the coupling $f: \mathbb{T}^{d} \times[0,+\infty) \rightarrow \mathbb{R}$ is continuous in both variables, increasing with respect to the second variable $m$, and there exist $q>1$ and $C_{1}$ such that

$$
\frac{1}{C_{1}}|m|^{q-1}-C_{1} \leq f(x, m) \leq C_{1}|m|^{q-1}+C_{1} \quad \forall m \geq 0 .
$$

Moreover we ask the following normalization condition to hold:

$$
f(x, 0)=0 \quad \forall x \in \mathbb{T}^{d} .
$$

We denote by $p$ the conjugate of $q: 1 / p+1 / q=1$.

(H2) (Conditions on the Hamiltonian) The Hamiltonian $H: \mathbb{T}^{d} \times \mathbb{R}^{d} \rightarrow \mathbb{R}$ is continuous in both variables, convex and differentiable in the second variable, with $D_{p} H$ continuous in both variables, and has a superlinear growth in the gradient variable: there exist $r>1$ and $C_{2}>0$ such that

$$
\frac{1}{r C_{2}}|\xi|^{r}-C_{2} \leq H(x, \xi) \leq \frac{C_{2}}{r}|\xi|^{r}+C_{2} \quad \forall(x, \xi) \in \mathbb{T}^{d} \times \mathbb{R}^{d} .
$$

We note for later use that the Fenchel conjugate $H^{*}$ of $H$ with respect to the second variable, i.e., $H^{*}(x, \xi):=\sup _{\zeta \in \mathbb{R}^{d}}\langle\xi, \zeta\rangle-H(x, \zeta)$, is continuous and satisfies similar inequalities

$$
\frac{1}{r^{\prime} C_{2}}|\xi|^{r^{\prime}}-C_{2} \leq H^{*}(x, \xi) \leq \frac{C_{2}}{r^{\prime}}|\xi|^{r^{\prime}}+C_{2} \quad \forall(x, \xi) \in \mathbb{T}^{d} \times \mathbb{R}^{d}
$$

where $r^{\prime}$ is the conjugate of $r: \frac{1}{r}+\frac{1}{r^{\prime}}=1$.

(H3) (Conditions on $A$ ) there exists a Lipschitz continuous map $\Sigma: \mathbb{T}^{d} \rightarrow$ $\mathbb{R}^{d \times D}$ such that $\Sigma \Sigma^{T}=A$ : let $C_{3}$ be a constant such that

$$
|\Sigma(x)-\Sigma(y)| \leq C_{3}|x-y| \quad \forall x, y \in \mathbb{T}^{d} .
$$

Moreover we suppose that

$$
\text { either } r \geq p \quad \text { or } \quad A \text { is constant. }
$$

We recall that $p$ is the conjugate of $q$.

(H4) (Conditions on the initial and terminal data) $\phi_{T}: \mathbb{T}^{d} \rightarrow \mathbb{R}$ is of class $\mathcal{C}^{2}$, while $m_{0}: \mathbb{T}^{d} \rightarrow \mathbb{R}$ is a $C^{1}$ positive density (namely $m_{0}>0$ and $\left.\int_{\mathbb{T}^{d}} m_{0} d x=1\right)$.

Condition (3) is just a normalization condition, which we may assume without loss of generality. Indeed, if all the conditions (H1)...(H4) but (3) hold, then one just needs to replace $f(x, m)$ by $f(x, m)-f(x, 0)$ and $H(x, p)$ by $H(x, p)-f(x, 0)$ : the new $H$ and $f$ still satisfy the above conditions (H1)...(H4) with (3). Note that assumption (H1) does not allow couplings which are not bounded below, in contrast with $[4,11]$. 
Let us set

$$
F(x, m)= \begin{cases}\int_{0}^{m} f(x, \tau) d \tau & \text { if } m \geq 0 \\ +\infty & \text { otherwise }\end{cases}
$$

Then $F$ is continuous on $\mathbb{T}^{d} \times(0,+\infty)$, differentiable and strictly convex in $m$ and satisfies

$$
\frac{1}{q C_{1}}|m|^{q}-C_{1} \leq F(x, m) \leq \frac{C_{1}}{q}|m|^{q}+C_{1} \quad \forall m \geq 0
$$

(changing the constant $C_{1}$ if necessary). Let $F^{*}$ be the Fenchel conjugate of $F$ with respect to the second variable. Note that $F^{*}(x, a)=0$ for $a \leq 0$ because $F(x, m)$ is nonnegative and equal to $+\infty$ for $m<0$. Moreover,

$$
\frac{1}{p C_{1}}|a|^{p}-C_{1} \leq F^{*}(x, a) \leq \frac{C_{1}}{p}|a|^{p}+C_{1} \quad \forall a \geq 0 .
$$

Some comments on the growth condition on the data are now in order. If $A$ is constant (as, for instance, in the first order case), we do not require any relation between the growth of $H$ and the growth of $F$ : both maps just need to be superlinear. This is an extension compared to $[3,4]$. The price to pay is that we can no longer guarantee the boundedness of solutions. When the diffusion depends on the space variable, we need the relation $r \geq p$, where $p$ is the growth of $F^{*}$ : this is required by a regularization procedure in Lemma 5.3. Note that our growth conditions prevent couplings $f$ with a slow growth; this is due to the possibly degenerate character of the diffusion, such restrictions are not needed in the uniformly parabolic case (see $[11-13,23])$.

\section{Basic estimates on solutions of Hamilton-Jacobi equations}

In this section we prove estimates in Lebesgue spaces for subsolutions of Hamilton-Jacobi equations of the form

$$
\left\{\begin{array}{l}
\text { (i) }-\partial_{t} \phi-A_{i j}(x) \partial_{i j} \phi+H(x, D \phi) \leq \alpha(t, x) \\
\text { (ii) } \phi(x, T) \leq \phi_{T}(x)
\end{array}\right.
$$

in terms of Lebesgue norms of $\alpha$ and $\phi_{T}$. We assume that (4) and (6) hold, and (10) is understood in the sense of distributions. This means that $D \phi \in L^{r}$ and, for any nonnegative test function $\zeta \in C_{c}^{\infty}\left((0, T] \times \mathbb{T}^{d}\right)$,

$$
\begin{aligned}
& -\int_{\mathbb{T}^{d}} \zeta(T) \phi_{T}+\int_{0}^{T} \int_{\mathbb{T}^{d}} \phi \partial_{t} \zeta+\langle D \zeta, A D \phi\rangle+\zeta\left(\partial_{i} A_{i j} \partial_{j} \phi+H(x, D \phi)\right) \\
& \quad \leq \int_{0}^{T} \int_{\mathbb{T}^{d}} \alpha \zeta .
\end{aligned}
$$

The estimates will be a consequence of the divergence structure of second order terms. 
Theorem 3.1. Assume that $\phi \in L^{r}\left((0, T) ; W^{1, r}\left(\mathbb{T}^{d}\right)\right)$ is a nonnegative function satisfying, in distributional sense,

$$
\left\{\begin{array}{l}
\left(\text { i) }-\partial_{t} \phi-\partial_{i}\left(A_{i j}(x) \partial_{j} \phi\right)+c_{0}|D \phi|^{r} \leq \alpha(t, x)\right. \\
\left(\text { ii) } \phi(x, T) \leq \phi_{T}(x)\right.
\end{array}\right.
$$

for some nonnegative, bounded Lipschitz matrix $A_{i j}$, and some $r>1, c_{0}>0$, $\alpha \in L^{p}\left((0, T) \times \mathbb{T}^{d}\right)$ and $\phi_{T} \in L^{\infty}\left(\mathbb{T}^{d}\right)$. Then, there exists a constant $C=$ $C\left(p, d, r, c_{0}, T,\|\alpha\|_{L^{p}\left((0, T) \times \mathbb{T}^{d}\right)},\left\|\phi_{T}\right\|_{L^{\eta}\left(\mathbb{T}^{d}\right)}\right)$ such that

$$
\|\phi\|_{L^{\infty}\left((0, T), L^{\eta}\left(\mathbb{T}^{d}\right)\right)}+\|\phi\|_{L^{\gamma}\left((0, T) \times \mathbb{T}^{d}\right)} \leq C
$$

where $\eta=\frac{d(r(p-1)+1)}{d-r(p-1)}$ and $\gamma=\frac{r p(1+d)}{d-r(p-1)}$ if $p<1+\frac{d}{r}$ and $\eta=\gamma=+\infty$ if $p>1+\frac{d}{r}$.

We note for later use that $\gamma>r$.

Proof. Up to a rescaling, we may assume that $c_{0}=1$. We first claim that, for any real function $g \in W^{1, \infty}(\mathbb{R})$ which is nondecreasing, and nonnegative in $\mathbb{R}_{+}$, we have

$$
\begin{gathered}
\int_{\mathbb{T}^{d}} G(\phi(\tau)) d x+\int_{\tau}^{T} \int_{\mathbb{T}^{d}}|D \phi|^{r} g(\phi) d x d t \\
\leq \int_{\tau}^{T} \int_{\mathbb{T}^{d}} \alpha g(\phi) d x d t+\int_{\mathbb{T}^{d}} G\left(\phi_{T}\right) d x
\end{gathered}
$$

for a.e. $\tau \in(0, T)$, where $G(r)=\int_{0}^{r} g(s) d s$.

There are several possible ways to justify (12), one is to use regularization.

We first extend $\phi$ to $(0, T+1] \times \mathbb{T}^{d}$ by defining $\phi=\phi_{T}$ on $[T, T+1]$. Then it still holds in the sense of distributions

$$
-\partial_{t} \phi-\partial_{i}\left(\tilde{A}_{i j}(t, x) \partial_{j} \phi\right)+|D \phi|^{r} \chi_{(0, T)} \leq \tilde{\alpha}(t, x)
$$

where $\tilde{A}_{i j}(t, x)=A_{i j}(x) \chi_{(0, T)}(t)$ and $\tilde{\alpha}(t, x)=\alpha(t, x) \chi_{(0, T)}(t)$. Let $\xi$ be a standard convolution kernel in $(t, x)$ defined on $\mathbb{R}^{d+1}$, with support in the unit ball of $\mathbb{R}^{d+1}$ and $\xi^{\epsilon}(t, x)=\xi((t, x) / \epsilon) /(\epsilon)^{d+1}, \xi^{\epsilon} \geq 0, \int_{\mathbb{R}^{d+1}} \xi^{\epsilon}(t, x) d t d x=1$, for all $\epsilon>0$. Let $\phi_{\epsilon}=\xi^{\epsilon} \star \phi$ and $\alpha_{\epsilon}=\xi^{\epsilon} \star \tilde{\alpha}$. Then $\phi_{\epsilon}, \alpha_{\epsilon}$ are $C^{\infty}$ and converge to $\phi, \alpha$ in their respective Lebesgue spaces. Convolving $\xi^{\epsilon}$ with (13) we obtain on $(\epsilon, T+1) \times \mathbb{T}^{d}$ :

$$
-\partial_{t} \phi_{\epsilon}-\partial_{i}\left(\tilde{A}_{i j}(t, x) \partial_{j} \phi_{\epsilon}\right)+\left|\xi_{\epsilon} \star(D \tilde{\phi})\right|^{r} \leq \alpha_{\epsilon}(t, x)+R_{\epsilon}
$$

where $D \tilde{\phi}=D \phi \chi_{(0, T)}$ and we used the fact that $D \phi \mapsto|D \phi|^{r}$ is convex, and where $R_{\epsilon}=-\partial_{i}\left(\tilde{A}_{i j}(t, x) \partial_{j} \phi_{\epsilon}\right)+\xi_{\epsilon} \star \partial_{i}\left(\tilde{A}_{i j}(t, x) \partial_{j} \phi\right)$.

Using the notation (cf. [9])

$$
\left[\xi^{\epsilon}, c\right](f):=\xi^{\epsilon} \star(c f)-c\left(\xi^{\epsilon} \star f\right)
$$

we can rewrite $R_{\epsilon}$ as $R_{\epsilon}=\left[\xi_{\epsilon}, \partial_{i} \tilde{A}_{i j}\right]\left(\partial_{j} \phi\right)+\left[\xi_{\epsilon}, \tilde{A}_{i j} \partial_{i}\right]\left(\partial_{j} \phi\right)$. Invoking [9, Lemma II.1], we have that $R_{\epsilon} \rightarrow 0$ in $L^{r}$, since $D \phi \in L^{r}$ and $A_{i j}$ is Lipschitz. 
Multiplying by $g\left(\phi_{\epsilon}\right)$ and integrating over $[\tau, T+\epsilon] \times \mathbb{T}^{d}$, for $\tau \in(\epsilon, T)$, it follows

$$
\begin{aligned}
& \int_{\mathbb{T}^{d}} G\left(\phi_{\epsilon}(\tau)\right) d x-\int_{\mathbb{T}^{d}} G\left(\phi_{\epsilon}(T+\epsilon)\right) d x+\int_{\tau}^{T} \int_{\mathbb{T}^{d}} A_{i j}(x) \partial_{j} \phi_{\epsilon} g^{\prime}\left(\phi_{\epsilon}\right) \partial_{i} \phi_{\epsilon} d x d t \\
& \quad+\int_{\tau}^{T+\epsilon} \int_{\mathbb{T}^{d}}\left|\xi_{\epsilon} \star(D \tilde{\phi})\right|^{r} g\left(\phi_{\epsilon}\right) d x d t \leq \int_{\tau}^{T+\epsilon} \int_{\mathbb{T}^{d}} g\left(\phi_{\epsilon}\right) \alpha_{\epsilon}(t, x) d x d t \\
& \quad+\int_{\tau}^{T+\epsilon} \int_{\mathbb{T}^{d}} g\left(\phi_{\epsilon}\right) R_{\epsilon} d x d t .
\end{aligned}
$$

Since $\int_{\tau}^{T} \int_{\mathbb{T}^{d}} A_{i j}(x) \partial_{j} \phi_{\epsilon} g^{\prime}\left(\phi_{\epsilon}\right) \partial_{i} \phi_{\epsilon} d x d t \geq 0$, and since $\phi_{\epsilon}(T+\epsilon)=\xi^{\epsilon} \star \phi_{T}$, we obtain

$$
\begin{gathered}
\int_{\mathbb{T}^{d}} G\left(\phi_{\epsilon}(\tau)\right) d x-\int_{\mathbb{T}^{d}} G\left(\xi^{\epsilon} \star \phi_{T}\right) d x+\int_{\tau}^{T+\epsilon} \int_{\mathbb{T}^{d}}\left|\xi_{\epsilon} \star(D \tilde{\phi})\right|^{r} g\left(\phi_{\epsilon}\right) d x d t \\
\leq \int_{\tau}^{T+\epsilon} \int_{\mathbb{T}^{d}} g\left(\phi_{\epsilon}\right) \alpha_{\epsilon}(t, x) d x d t+\int_{\tau}^{T+\epsilon} \int_{\mathbb{T}^{d}} g\left(\phi_{\epsilon}\right) R_{\epsilon} d x d t .
\end{gathered}
$$

Since $g$ is bounded, while $R_{\epsilon}$ and $\alpha_{\epsilon}$ converge in $L^{r}\left((0, T) \times \mathbb{T}^{d}\right)$ and in $L^{p}\left((0, T) \times \mathbb{T}^{d}\right)$ respectively, we can pass to the limit as $\epsilon$ goes to zero and for almost every $\tau$ we get (12).

Now we proceed with the desired estimate. First we observe that, up to replacing $g(r)$ with $g(r \wedge k)$, we can assume that $\phi$ is bounded and that $g$ may be any $C^{1}$ function. In particular, we take $g(\phi)=\phi^{(\sigma-1) r}$ for $\sigma>1$, obtaining

$$
\begin{gathered}
\frac{1}{(\sigma-1) r+1} \int_{\mathbb{T}^{d}} \phi(\tau)^{(\sigma-1) r+1} d x+\frac{1}{\sigma^{r}} \int_{\tau}^{T} \int_{\mathbb{T}^{d}}\left|D \phi^{\sigma}\right|^{r} d x d t \\
\leq \int_{\tau}^{T} \int_{\mathbb{T}^{d}} \alpha \phi^{(\sigma-1) r} d x d t+\frac{1}{(\sigma-1) r+1} \int_{\mathbb{T}^{d}} \phi_{T}^{(\sigma-1) r+1} d x .
\end{gathered}
$$

Let us denote henceforth by $c$ possibly different constants only depending on $r, \sigma, d$ and $T$. By arbitrariness of $\tau$, the previous inequality implies

$$
\begin{gathered}
\left.\left\|\phi^{\sigma}\right\|_{L^{\infty}((0, T) ; L}^{\frac{(\sigma-1) r+1}{\sigma}}+\left\|D \phi^{\sigma}\right\|_{L^{r}\left((0, T) \times \mathbb{T}^{d}\right)}^{r}\left(\mathbb{T}^{d}\right)\right) \\
\leq c \int_{0}^{T} \int_{\mathbb{T}^{d}} \alpha \phi^{(\sigma-1) r} d x d t+c \int_{\mathbb{T}^{d}} \phi_{T}^{(\sigma-1) r+1} d x .
\end{gathered}
$$

On the other hand, by interpolation we have (see e.g. [8, Proposition 3.1, Chapter 1])

$$
\|v\|_{L^{q}\left((0, T) \times \mathbb{T}^{d}\right)}^{q} \leq c\|v\|_{L^{\infty}\left((0, T) ; L^{\eta}\left(\mathbb{T}^{d}\right)\right)}^{\frac{\eta r}{d}}\|D v\|_{L^{r}\left((0, T) \times \mathbb{T}^{d}\right)}^{r} \quad \text { where } q=r \frac{d+\eta}{d}
$$

for any $v \in L^{r}\left((0, T) ; W^{1, r}\left(\mathbb{T}^{d}\right)\right)$ such that $\int_{\mathbb{T}^{d}} v(t) d x=0$ a.e. in $(0, T)$. So we deduce that 


$$
\begin{aligned}
\left\|\phi^{\sigma}\right\|_{L^{q}\left((0, T) \times \mathbb{T}^{d}\right)}^{q} \leq & c\left\{\int_{0}^{T} \int_{\mathbb{T}^{d}} \alpha \phi^{(\sigma-1) r} d x d t+\int_{\mathbb{T}^{d}} \phi_{T}^{(\sigma-1) r+1} d x\right\}^{1+\frac{r}{d}} \\
& +c \int_{0}^{T}\left(\int_{\mathbb{T}^{d}} \phi(t)^{\sigma} d x\right)^{q} d t
\end{aligned}
$$

for $\eta=\frac{(\sigma-1) r+1}{\sigma}$ and $q=r \frac{\eta+d}{d}$. We choose $\sigma$ such that

$$
\sigma q=(\sigma-1) r p^{\prime}
$$

and therefore, by Hölder inequality, we conclude

$$
\begin{aligned}
\left\|\phi^{\sigma}\right\|_{L^{q}\left((0, T) \times \mathbb{T}^{d}\right)}^{q} \leq & c\|\alpha\|_{L^{p}\left((0, T) \times \mathbb{T}^{d}\right)}^{1+\frac{r}{d}}\left\|\phi^{\sigma}\right\|_{L^{q}\left((0, T) \times \mathbb{T}^{d}\right)}^{\frac{q}{p^{\prime}\left(1+\frac{r}{d}\right)}} \\
& +c\left(\int_{\mathbb{T}^{d}} \phi_{T}^{(\sigma-1) r+1} d x\right)^{1+\frac{r}{d}}+c \int_{0}^{T}\left(\int_{\mathbb{T}^{d}} \phi(t)^{\sigma} d x\right)^{q} d t .
\end{aligned}
$$

Since $\int_{\mathbb{T}^{d}} \phi(t) d x$ is estimated in terms of $\|\alpha\|_{L^{1}\left((0, T) \times \mathbb{T}^{d}\right)}$ and $\left\|\phi_{T}\right\|_{L^{1}\left(\mathbb{T}^{d}\right)}$, the last term can be absorbed into the left-hand side up to a constant $C=$ $C\left(\|\alpha\|_{L^{1}\left((0, T) \times \mathbb{T}^{d}\right)},\left\|\phi_{T}\right\|_{L^{1}\left(\mathbb{T}^{d}\right)}\right)$. Moreover, since $p<1+\frac{d}{r}$, we have $\frac{q}{p^{\prime}}\left(1+\frac{r}{d}\right)<$ $q$. Hence we end up with an estimate

$$
\left\|\phi^{\sigma}\right\|_{L^{q}\left((0, T) \times \mathbb{T}^{d}\right)}^{q} \leq C\left(\|\alpha\|_{L^{p}\left((0, T) \times \mathbb{T}^{d}\right)},\left\|\phi_{T}\right\|_{L^{(\sigma-1) r+1}\left(\mathbb{T}^{d}\right)}\right) .
$$

Computing the value of $\sigma$ in terms of $r$ and $p$ we get

$$
q \sigma=\frac{r p(1+d)}{d-r(p-1)} \quad \text { and } \quad(\sigma-1) r+1=\frac{d(r(p-1)+1)}{d-r(p-1)}
$$

so the first part of the Theorem is proved.

Finally, we prove the $L^{\infty}$ estimate by using a strategy which goes back to [24]. To this purpose, we replace $\phi$ with $\phi-k$ and use (12) with $g(s)=\left(s_{+}\right)^{r^{\prime}}$; for any $k \geq\left\|\phi_{T}\right\|_{L^{\infty}\left(\mathbb{T}^{d}\right)}$ we obtain

$$
\int_{\mathbb{T}^{d}}\left[(\phi-k)_{+}(\tau)\right]^{\sigma+1} d x+\int_{\tau}^{T} \int_{\mathbb{T}^{d}}\left|D(\phi-k)_{+}^{\sigma}\right|^{r} d x d t \leq \int_{\tau}^{T} \int_{\mathbb{T}^{d}} \alpha(\phi-k)_{+}^{\sigma} d x d t
$$

with $\sigma=r^{\prime}$. Using as before the embedding (14) we get

$$
\begin{aligned}
\left\|(\phi-k)_{+}^{\sigma}\right\|_{L^{q}\left((0, T) \times \mathbb{T}^{d}\right) \leq}^{q} \leq & \left\{\int_{0}^{T} \int_{\mathbb{T}^{d}} \alpha(\phi-k)_{+}^{\sigma} d x d t\right\}^{1+\frac{r}{d}} \\
& +c \int_{0}^{T}\left(\int_{\mathbb{T}^{d}}(\phi-k)_{+}^{\sigma} d x\right)^{q} d t \\
\leq & c\left\{\int_{0}^{T} \int_{\mathbb{T}^{d}} \alpha(\phi-k)_{+}^{\sigma} d x d t\right\}^{1+\frac{r}{d}} \\
& +c \int_{0}^{T}|\{x: \phi(t)>k\}|^{q-1} \int_{\mathbb{T}^{d}}(\phi-k)_{+}^{\sigma q} d x d t,
\end{aligned}
$$

where, using that $\sigma=r^{\prime}$, we have

$$
q=r \frac{\frac{\sigma+1}{\sigma}+d}{d}=\frac{r}{d}\left(d+2-\frac{1}{r}\right) .
$$


Notice that $|\{x: \phi(t)>k\}|$ is uniformly small provided $k$ is large, only depending on $\|\alpha\|_{L^{1}}$ and $\left\|\phi_{T}\right\|_{L^{1}}$. Therefore, absorbing the last term in the left-hand side we deduce

$$
\left\|(\phi-k)_{+}^{\sigma}\right\|_{L^{q}\left((0, T) \times \mathbb{T}^{d}\right)}^{q} \leq c\left\{\int_{0}^{T} \int_{\mathbb{T}^{d}} \alpha(\phi-k)_{+}^{\sigma} d x d t\right\}^{1+\frac{r}{d}}
$$

for some $c=c\left(\|\alpha\|_{L^{1}\left((0, T) \times \mathbb{T}^{d}\right)},\left\|\phi_{T}\right\|_{L^{1}\left(\mathbb{T}^{d}\right)}\right)$. One can check that, since $r>1$, (15) implies $q>1+\frac{r}{d}$ and, in particular, $\frac{1}{q}+\frac{1}{p}<1$. Thus, by Hölder inequality we get

$$
\begin{aligned}
& \left\|(\phi-k)_{+}^{\sigma}\right\|_{L^{q}\left((0, T) \times \mathbb{T}^{d}\right)}^{q} \\
& \quad \leq c\left\|(\phi-k)_{+}^{\sigma}\right\|_{L^{q}\left((0, T) \times \mathbb{T}^{d}\right)}^{1+\frac{r}{d}}\|\alpha\|_{L^{p}\left((0, T) \times \mathbb{T}^{d}\right)}^{1+\frac{r}{d}}\left|A_{k}\right|^{\left(1-\frac{1}{q}-\frac{1}{p}\right)\left(1+\frac{r}{d}\right)},
\end{aligned}
$$

where $A_{k}:=\{(t, x): \phi(t, x)>k\}$. Since, for any $h>k$ we have

$$
\int_{0}^{T} \int_{\mathbb{T}^{d}}(\phi-k)_{+}^{\sigma q} d x d t \geq\left|A_{h}\right|(h-k)^{\sigma q},
$$

we end up with the inequality

$$
\begin{aligned}
\left|A_{h}\right|^{1-\frac{1}{q}\left(1+\frac{r}{d}\right)}(h-k)^{\sigma q-\sigma\left(1+\frac{r}{d}\right)} & \leq\left\|(\phi-k)_{+}^{\sigma}\right\|_{L^{q}\left((0, T) \times \mathbb{T}^{d}\right)}^{q-\left(1+\frac{r}{d}\right)} \\
& \leq c\|\alpha\|_{L^{p}\left((0, T) \times \mathbb{T}^{d}\right)}^{1+\frac{r}{d}}\left|A_{k}\right|^{\left(1-\frac{1}{q}-\frac{1}{p}\right)\left(1+\frac{r}{d}\right)}
\end{aligned}
$$

which means that

$$
\left|A_{h}\right| \leq C \frac{\left|A_{k}\right|^{\beta}}{(h-k)^{\delta}} \quad \forall h>k \geq\left\|\phi_{T}\right\|_{L^{\infty}\left(\mathbb{T}^{d}\right)}
$$

for some $C=C\left(\|\alpha\|_{L^{p}\left((0, T) \times \mathbb{T}^{d}\right)}\right)$, some $\delta>0$ and with $\beta=\frac{\left(1-\frac{1}{q}-\frac{1}{p}\right)\left(1+\frac{r}{d}\right)}{1-\frac{1}{q}\left(1+\frac{r}{d}\right)}$. One can check that $\beta>1$ since $p>1+\frac{d}{r}$. Therefore, by a classical iteration lemma (see e.g. [24]), it follows that $\left|A_{k_{0}}\right|=0$ for some (explicit) $k_{0}>0$, which in particular implies the desired bound in terms of $\|\alpha\|_{L^{p}\left((0, T) \times \mathbb{T}^{d}\right)}$ and $\left\|\phi_{T}\right\|_{L^{\infty}\left(\mathbb{T}^{d}\right)}$.

Remark 3.2. The assumption that $\phi$ is nonnegative can be dropped and in this case the estimates are given on $\phi_{+}$; indeed, if $\phi$ satisfies $(11)$, then $\phi_{+}$ also does. This can be seen in the previous proof by taking $g=g\left(r_{+}\right)$, with $g(0)=0$.

Let us also stress that the Lipschitz continuity of the matrix $A$ was only used to recover the estimate from the distributional formulation (namely, to be sure that $\phi$ is limit of solutions of smooth approximating problems). The constant $C$ of the estimate, however, does not depend on $A$ in any way; in particular, the estimate will hold uniformly for any viscous approximation to possibly less regular matrices.

As a corollary, we deduce the following result for problem (10). 
Theorem 3.3. Assume that (4) and (6) hold true and let $\phi$ satisfy (10) with $\alpha \in L^{p}\left((0, T) \times \mathbb{T}^{d}\right), \phi_{T} \in L^{\infty}\left(\mathbb{T}^{d}\right)$. Then, $\phi_{+}$satisfies the estimates of Theorem 3.1. In particular, if $\phi$ is bounded below, we have

$$
\|\phi\|_{L^{\infty}\left((0, T), L^{\eta}\left(\mathbb{T}^{d}\right)\right)}+\|\phi\|_{L^{\gamma}\left((0, T) \times \mathbb{T}^{d}\right)} \leq C
$$

where $\eta=\frac{d(r(p-1)+1)}{d-r(p-1)}$ and $\gamma=\frac{r p(1+d)}{d-r(p-1)}$ if $p<1+\frac{d}{r}$ and $\eta=\gamma=+\infty$ if $p>1+\frac{d}{r}$, with a constant $C$ depending on $T, p, d, r, C_{2}, C_{3}$ [appearing in (4) and (6)] and on $\|\alpha\|_{L^{p}\left((0, T) \times \mathbb{T}^{d}\right)},\left\|\phi_{T}\right\|_{L^{\eta}\left(\mathbb{T}^{d}\right)}$ and $\left\|\phi_{-}\right\|_{L^{\infty}\left(\mathbb{T}^{d}\right)}$.

\section{Two optimization problems}

Mean field games systems with local coupling can be studied as an optimality condition between two problems in duality.

The first optimization problem is described as follows: let us denote by $\mathcal{K}_{0}$ the set of maps $\phi \in \mathcal{C}^{2}\left([0, T] \times \mathbb{T}^{d}\right)$ such that $\phi(T, x)=\phi_{T}(x)$ and define, on $\mathcal{K}_{0}$, the functional

$$
\begin{aligned}
\mathcal{A}(\phi)= & \int_{0}^{T} \int_{\mathbb{T}^{d}} F^{*}\left(x,-\partial_{t} \phi(t, x)-A_{i j} \partial_{i j} \phi+H(x, D \phi(t, x))\right) d x d t \\
& -\int_{\mathbb{T}^{d}} \phi(0, x) d m_{0}(x) .
\end{aligned}
$$

Then the problem consists in optimizing

$$
\inf _{\phi \in \mathcal{K}_{0}} \mathcal{A}(\phi) .
$$

For the second optimization problem, let $\mathcal{K}_{1}$ be the set of pairs $(m, w) \in$ $L^{1}\left((0, T) \times \mathbb{T}^{d}\right) \times L^{1}\left((0, T) \times \mathbb{T}^{d}, \mathbb{R}^{d}\right)$ such that $m(t, x) \geq 0$ a.e., with $\int_{\mathbb{T}^{d}}$ $m(t, x) d x=1$ for a.e. $t \in(0, T)$, and which satisfy in the sense of distributions the continuity equation

$$
\partial_{t} m-\partial_{i j}\left(A_{i j}(x) m\right)+\operatorname{div}(w)=0 \text { in }(0, T) \times \mathbb{T}^{d}, \quad m(0)=m_{0} .
$$

On the set $\mathcal{K}_{1}$, let us define the following functional

$$
\begin{aligned}
\mathcal{B}(m, w)= & \int_{0}^{T} \int_{\mathbb{T}^{d}} m(t, x) H^{*}\left(x,-\frac{w(t, x)}{m(t, x)}\right)+F(x, m(t, x)) d x d t \\
& +\int_{\mathbb{T}^{d}} \phi_{T}(x) m(T, x) d x
\end{aligned}
$$

where, for $m(t, x)=0$, we impose that

$$
m(t, x) H^{*}\left(x,-\frac{w(t, x)}{m(t, x)}\right)=\left\{\begin{array}{ll}
+\infty & \text { if } w(t, x) \neq 0 \\
0 & \text { if } w(t, x)=0
\end{array} .\right.
$$

Since $H^{*}$ and $F$ are bounded from below and $m \geq 0$ a.e., the first integral in $\mathcal{B}(m, w)$ is well defined in $\mathbb{R} \cup\{+\infty\}$.

In order to give a meaning to the last integral $\int_{\mathbb{T}^{d}} \phi_{T}(x) m(T, x) d x$, we use the standard fact that the measure $m(t)$ is actually defined for any $t$ (see Lemma 4.1 below). Indeed, let us define $v(t, x)=-\frac{w(t, x)}{m(t, x)}$ if $m(t, x)>0$ and 
$v(t, x)=0$ otherwise. Thanks to the growth of $H^{*}$ (see $\left.(5)\right), \mathcal{B}(m, w)$ is infinite if $m|v|^{r^{\prime}} \notin L^{1}(d x d t)$. Therefore, we can assume without loss of generality that $m|v|^{r^{\prime}} \in L^{1}(d x d t)$, or, equivalently, that $v \in L^{r^{\prime}}(m d x d t)$. In this case equation (18) can be rewritten as a Kolmogorov equation

$$
\partial_{t} m-\partial_{i j}\left(A_{i j}(x) m\right)-\operatorname{div}(m v)=0 \text { in }(0, T) \times \mathbb{T}^{d}, \quad m(0)=m_{0} .
$$

Lemma 4.1. There is a constant $C$, depending on $\|-w / m\|_{L_{m}^{r^{\prime}}}$ and on $\|A\|_{\infty}$, such that

$$
\mathbf{d}_{\mathbf{1}}\left(\mathbf{m}(\mathbf{t}), \mathbf{m}\left(\mathbf{t}^{\prime}\right)\right) \leq \mathbf{C}\left|\mathbf{t}-\mathbf{t}^{\prime}\right|^{\frac{1}{2} \vee \frac{1}{\mathbf{r}}} \quad \forall \mathbf{t}, \mathbf{t}^{\prime} \in[\mathbf{0}, \mathbf{T}]
$$

For the sake of completeness, we give the proof here.

Proof. We first extend the pair $(m, w)$ to $[-1, T] \times \mathbb{T}^{d}$ by defining $m=$ $m_{0}$ on $[-1,0]$ and $w(s, x)=0$ for $(s, x) \in(-1,0) \times \mathbb{T}^{d}$. Note that $\partial_{t} m-$ $\partial_{i j}\left(\tilde{A}_{i j}(t, x) m\right)+\operatorname{div}(w)=0$ holds in the sense of distributions on $(-1, T) \times \mathbb{T}^{d}$, where $\tilde{A}_{i j}(t, x)=A_{i j}(x)$ if $t \in(0, T)$ and $\tilde{A}_{i j}(t, x)=0$ otherwise. Let $\xi$ be a standard convolution kernel in $(t, x)$, with a support contained in the unit ball of $\mathbb{R}^{d+1}$ and $\xi^{\epsilon}(t, x)=\xi((t, x) / \epsilon) /(\epsilon)^{d+1}, \xi^{\epsilon} \geq 0, \int_{\mathbb{R}^{d+1}} \xi^{\epsilon}(t, x) d t d x=1$, for all $\epsilon>0$. Let $m_{\epsilon}=\xi^{\epsilon} \star m$ and $w_{\epsilon}=\xi^{\epsilon} \star w$. Then $m_{\epsilon}, w_{\epsilon}$ are $C^{\infty}$ and $\int_{\mathbb{T}^{d}} m_{\epsilon}(t, x) d x=1$ for all $t \in(-1+\epsilon, T-\epsilon)$ and $\epsilon>0$ small enough.

Convolving $\xi^{\epsilon}$ with (18), we obtain

$$
\partial_{t} m_{\epsilon}-\partial_{i j}\left(\xi^{\epsilon} *\left(\tilde{A}_{i j}(t, x) m\right)\right)+\operatorname{div}\left(w_{\epsilon}\right)=0 \text { in }(-1 / 2, T-\epsilon) \times \mathbb{T}^{d},
$$

with

$$
m_{\epsilon}(-1 / 2, x)=\int_{\mathbb{R}} \int_{\mathbb{T}^{d}} \xi^{\epsilon}(s, x-y) m_{0}(y) d y d s .
$$

The equation can be rewritten as

$$
\left.\partial_{t} m_{\epsilon}-\partial_{i j}\left(\tilde{A}_{i j}^{\epsilon}(t, x) m_{\epsilon}\right)\right)-\operatorname{div}\left(m_{\epsilon} v_{\epsilon}\right)=0 \quad \text { in }(-1 / 2, T-\epsilon) \times \mathbb{T}^{d}
$$

where $\tilde{A}_{i j}^{\epsilon}=\frac{\xi^{\epsilon} \star\left(\tilde{A}_{i j} m\right)}{m_{\epsilon}}$ and $v_{\epsilon}=-\frac{w_{\epsilon}}{m_{\epsilon}}$.

Let us consider the following stochastic differential equations defined for all $\epsilon>0$

$$
\left\{\begin{array}{l}
d X_{t}^{\epsilon}=v_{\epsilon}\left(t, X_{t}^{\epsilon}\right) d t+\Sigma_{\epsilon}\left(X_{t}^{\epsilon}\right) d B_{t}^{\epsilon} \quad t \in[-1 / 2, T-\epsilon] \\
X_{-1 / 2}^{\epsilon}=Z_{-1 / 2}^{\epsilon}
\end{array},\right.
$$

where $d B_{t}^{\epsilon}$ is a standard $d$-dimensional Brownian motion over some probability space $(\Omega, \mathcal{A}, \mathbb{P}), \Sigma_{\epsilon} \Sigma_{\epsilon}^{T}=\tilde{A}^{\epsilon}$, and the initial condition $Z_{-1 / 2}^{\epsilon} \in L^{1}\left(\mathbb{T}^{d}\right)$ is random, independent of $\left(B_{t}^{\epsilon}\right)$ and with law $m_{\epsilon}(-1 / 2, \cdot)$.

For all $\epsilon>0$, the vector field $v_{\epsilon}$ is continuous, uniformly Lipschitz continuous in space and bounded. Therefore, there exists a unique solution to (21). Moreover, as a consequence of Ito's formula, we have that, if the density $\mathcal{L}\left(Z_{0}^{\epsilon}\right)=\xi^{\epsilon} \star m_{0}$, then $m_{\epsilon}(t)=\mathcal{L}\left(X_{t}^{\epsilon}\right)$ solves $(20)$ in the sense of distributions.

Let $\mathbf{d}_{\mathbf{1}}$ be the Kantorovich-Rubinstein distance on $P\left(\mathbb{T}^{d}\right)$ and $\gamma_{\epsilon} \in$ $\Pi\left(m_{\epsilon}(t), m_{\epsilon}(s)\right)$ the law of the pair $\left(X_{t}^{\epsilon}, X_{s}^{\epsilon}\right)$ for $0 \leq s<t \leq T$, where $\Pi\left(m_{\epsilon}(t), m_{\epsilon}(s)\right)$ is the set of Borel probability measures $\mu$ on $\mathbb{T}^{d} \times \mathbb{T}^{d}$ such 
that $\mu\left(A \times \mathbb{T}^{d}\right)=m_{\epsilon}(t, A)$ and $\mu\left(\mathbb{T}^{d} \times A\right)=m_{\epsilon}(s, A)$ for any Borel set $A \in \mathbb{T}^{d}$. We have

$$
\mathbf{d}_{\mathbf{1}}\left(m_{\epsilon}(t), m_{\epsilon}(s)\right) \leq \int_{\mathbb{T}^{d} \times \mathbb{T}^{d}}|x-y| d \gamma_{\epsilon}(x, y)=\mathbb{E}\left[\left|X_{t}^{\epsilon}-X_{s}^{\epsilon}\right|\right] .
$$

Moreover,

$$
\begin{aligned}
\mathbb{E}\left[\left|X_{t}^{\epsilon}-X_{s}^{\epsilon}\right|\right] & \leq \mathbb{E}\left[\int_{s}^{t}\left|v_{\epsilon}\left(\tau, X_{\tau}^{\epsilon}\right)\right| d \tau\right]+\mathbb{E}\left[\left|\int_{s}^{t} \Sigma_{\epsilon}\left(X_{\tau}^{\epsilon}\right) d B_{\tau}\right|\right] \\
& \leq \int_{s}^{t} \int_{\mathbb{T}^{d}}\left|v_{\epsilon}(\tau, x)\right| m_{\epsilon}(\tau, x) d x d \tau+\left(\mathbb{E}\left[\int_{s}^{t} \Sigma_{\epsilon} \Sigma_{\epsilon}^{*}\left(X_{\tau}^{\epsilon}\right) d \tau\right]\right)^{1 / 2} \\
& \leq \int_{s}^{t} \int_{\mathbb{T}^{d}}\left|v_{\epsilon}(\tau, x)\right| m_{\epsilon}(\tau, x) d x d \tau+\|A\|_{\infty} C|t-s|^{\frac{1}{2}}
\end{aligned}
$$

Recalling the definition of $v_{\epsilon}$, we have that $m_{\epsilon}\left|v_{\epsilon}\right|^{r^{\prime}}=\frac{\left|w_{\epsilon}\right|^{r^{\prime}}}{m_{\epsilon}^{r^{\prime}}-1}$ belongs to $L^{1}\left([0, T] \times \mathbb{T}^{d}\right)$ for all $\epsilon>0$. Indeed, the function $(m, w) \mapsto \frac{|w|^{r^{\prime}}}{m^{r^{\prime}-1}}$ is convex and $\frac{|w|^{r^{\prime}}}{m^{r^{\prime}-1}}$ belongs to $L^{1}\left([0, T] \times \mathbb{T}^{d}\right)$. Thus

$$
\int_{0}^{T} \int_{\mathbb{T}^{d}} \frac{\left|\xi^{\epsilon} \star w\right|^{r^{\prime}}}{\left(\xi^{\epsilon} \star m\right)^{r^{\prime}-1}} d x d \tau \leq \int_{0}^{T} \int_{\mathbb{T}^{d}} \xi^{\epsilon} \star\left(\frac{|w|^{r^{\prime}}}{m^{r^{\prime}-1}}\right) d x d \tau \leq\left\|\frac{|w|^{r^{\prime}}}{m^{r^{\prime}-1}}\right\|_{1} .
$$

Therefore, using Hölder inequality,

$$
\begin{aligned}
\mathbf{d}_{\mathbf{1}}\left(m_{\epsilon}(t), m_{\epsilon}(s)\right) \leq & \left(\int_{s}^{t} \int_{\mathbb{T}^{d}}\left|v_{\epsilon}(\tau, x)\right|^{r^{\prime}} m_{\epsilon}(\tau, x) d x d \tau\right)^{\frac{1}{r^{\prime}}} \\
& \times\left(\int_{s}^{t} \int_{\mathbb{T}^{d}} m_{\epsilon}(\tau, x) d x d \tau\right)^{\frac{1}{r}}+\|A\|_{\infty} C|t-s|^{\frac{1}{2}} \\
\leq & \left\|\frac{|w|^{r^{\prime}}}{m^{r^{\prime}-1}}\right\|_{1}^{\frac{1}{r^{\prime}}}|t-s|^{\frac{1}{r}}+\|A\|_{\infty}|t-s|^{\frac{1}{2}} .
\end{aligned}
$$

Letting $\epsilon \rightarrow 0$ we have $m_{\epsilon} \rightarrow m$ in $L^{1}\left([0, T] \times \mathbb{T}^{d}\right)$ and for a.e. $\tau \in[0, T]$, $m_{\epsilon}(\tau) \rightarrow m(\tau)$ in $L^{1}\left(\mathbb{T}^{d}\right)$, moreover for a.e. $0 \leq s<t \leq T$

$$
\lim _{\epsilon \rightarrow 0} \mathbf{d}_{\mathbf{1}}\left(m_{\epsilon}(t), m_{\epsilon}(s)\right)=\mathbf{d}_{\mathbf{1}}(m(t), m(s)) .
$$

Thus for a.e. $0 \leq s<t \leq T$

$$
\mathbf{d}_{\mathbf{1}}(m(t), m(s)) \leq C|t-s|^{\frac{1}{r}}+\|A\|_{\infty}|t-s|^{\frac{1}{2}} .
$$

The second optimal control problem is the following:

$$
\inf _{(m, w) \in \mathcal{K}_{1}} \mathcal{B}(m, w) .
$$


Lemma 4.2. We have

$$
\inf _{\phi \in \mathcal{K}_{0}} \mathcal{A}(\phi)=-\min _{(m, w) \in \mathcal{K}_{1}} \mathcal{B}(m, w) .
$$

Moreover, the minimum in the right-hand side is achieved by a unique pair $(m, w) \in \mathcal{K}_{1}$ satisfying $(m, w) \in L^{q}\left((0, T) \times \mathbb{T}^{d}\right) \times L^{\frac{r^{\prime} q}{r^{\prime}+q-1}}\left((0, T) \times \mathbb{T}^{d}\right)$.

Remark 4.3. Note that $\frac{r^{\prime} q}{r^{\prime}+q-1}>1$ because $r^{\prime}>1$ and $q>1$.

Proof. The strategy of proof - which is very close to the corresponding one in [3-5] - consists in applying the Fenchel-Rockafellar duality theorem (cf. e.g., [10]). In order to do so, it is better to reformulate the first optimization problem $(17)$ in a more suitable form. Let $E_{0}=\mathcal{C}^{2}\left([0, T] \times \mathbb{T}^{d}\right)$ and $E_{1}=\mathcal{C}^{0}([0, T] \times$ $\left.\mathbb{T}^{d}, \mathbb{R}\right) \times \mathcal{C}^{0}\left([0, T] \times \mathbb{T}^{d}, \mathbb{R}^{d}\right)$. We define on $E_{0}$ the functional

$$
\mathcal{F}(\phi)=-\int_{\mathbb{T}^{d}} m_{0}(x) \phi(0, x) d x+\chi_{S}(\phi),
$$

where $\chi_{S}$ is the characteristic function of the set $S=\left\{\phi \in E_{0}, \phi(T, \cdot)=\phi_{T}\right\}$, i.e., $\chi_{S}(\phi)=0$ if $\phi \in S$ and $+\infty$ otherwise. For $(a, b) \in E_{1}$, we define

$$
\mathcal{G}(a, b)=\int_{0}^{T} \int_{\mathbb{T}^{d}} F^{*}(x,-a(t, x)+H(x, b(t, x))) d x d t .
$$

The functional $\mathcal{F}$ is convex and lower semi-continuous on $E_{0}$ while $\mathcal{G}$ is convex and continuous on $E_{1}$. Let $\Lambda: E_{0} \rightarrow E_{1}$ be the bounded linear operator defined by $\Lambda(\phi)=\left(\partial_{t} \phi+A_{i j} \partial_{i j} \phi, D \phi\right)$. We can observe that

$$
\inf _{\phi \in \mathcal{K}_{0}} \mathcal{A}(\phi)=\inf _{\phi \in E_{0}}\{\mathcal{F}(\phi)+\mathcal{G}(\Lambda(\phi))\} .
$$

It is easy to verify that the qualification hypothesis, that ensures the stability of the above optimization problem, holds. Indeed, there is a map $\phi$ such that $\mathcal{F}(\phi)<+\infty$ and such that $\mathcal{G}$ is continuous at $\Lambda(\phi)$ : it is enough to take $\phi(t, x)=\phi_{T}(x)$.

Therefore we can apply the Fenchel-Rockafellar duality theorem, which states that

$$
\inf _{\phi \in E_{0}}\{\mathcal{F}(\phi)+\mathcal{G}(\Lambda(\phi))\}=\max _{(m, w) \in E_{1}^{\prime}}\left\{-\mathcal{F}^{*}\left(\Lambda^{*}(m, w)\right)-\mathcal{G}^{*}(-(m, w))\right\}
$$

where $E_{1}^{\prime}$ is the dual space of $E_{1}$, i.e., the set of vector valued Radon measures $(m, w)$ over $[0, T] \times \mathbb{T}^{d}$ with values in $\mathbb{R} \times \mathbb{R}^{d}, E_{0}^{\prime}$ is the dual space of $E_{0}$, $\Lambda^{*}: E_{1}^{\prime} \rightarrow E_{0}^{\prime}$ is the dual operator of $\Lambda$ and $\mathcal{F}^{*}$ and $\mathcal{G}^{*}$ are the convex conjugates of $\mathcal{F}$ and $\mathcal{G}$ respectively. By a direct computation we have

$$
\begin{aligned}
\mathcal{F}^{*} & \left(\Lambda^{*}(m, w)\right) \\
& = \begin{cases}\int_{\mathbb{T}^{d}} \phi_{T}(x) d m(T, x) & \text { if } \partial_{t} m-\partial_{i j}\left(A_{i j} m\right)+\operatorname{div}(w)=0, m(0)=m_{0} \\
+\infty & \text { otherwise }\end{cases}
\end{aligned}
$$

where the equation $\partial_{t} m-\partial_{i j}\left(A_{i j} m\right)+\operatorname{div}(w)=0, m(0)=m_{0}$ holds in the sense of distributions. Following [3], we have $\mathcal{G}^{*}(m, w)=+\infty$ if $(m, w) \notin L^{1}$ and, if $(m, w) \in L^{1}$, 


$$
\mathcal{G}^{*}(m, w)=\int_{0}^{T} \int_{\mathbb{T}^{d}} K^{*}(x, m(t, x), w(t, x)) d t d x
$$

where

$$
K^{*}(x, m, w)= \begin{cases}F(x,-m)-m H^{*}\left(x,-\frac{w}{m}\right) & \text { if } m>0 \\ 0 & \text { if } m=0, w=0 \\ +\infty & \text { otherwhise }\end{cases}
$$

is the convex conjugate of

$$
K(x, a, b)=F^{*}(x,-a+H(x, b)) \quad \forall(x, a, b) \in \mathbb{T}^{d} \times \mathbb{R} \times \mathbb{R}^{d} .
$$

Therefore

$$
\begin{aligned}
& \max _{(m, w) \in E_{1}^{\prime}}\left\{-\mathcal{F}^{*}\left(\Lambda^{*}(m, w)\right)-\mathcal{G}^{*}(-(m, w))\right\} \\
& =\max \left\{\int_{0}^{T} \int_{\mathbb{T}^{d}}-F(x, m)-m H^{*}\left(x,-\frac{w}{m}\right) d t d x-\int_{\mathbb{T}^{d}} \phi_{T}(x) m(T, x) d x\right\}
\end{aligned}
$$

where the last maximum is taken over the $L^{1}$ maps $(m, w)$ such that $m \geq 0$ a.e. and

$$
\partial_{t} m-\partial_{i j}\left(A_{i j} m\right)+\operatorname{div}(w)=0, m(0)=m_{0}
$$

holds in the sense of distributions. Since $\int_{\mathbb{T} d} m_{0}=1$, it follows that $\int_{\mathbb{T}^{d}} m(t)=$ 1 for any $t \in[0, T]$. Thus the pair $(m, w)$ belongs to the set $\mathcal{K}_{1}$ and the first part of the statement is proved.

Take now an optimal $(m, w) \in \mathcal{K}_{1}$ in the above system. Observe that due to optimality we have $w(t, x)=0$ for all $(t, x) \in[0, T] \times \mathbb{T}^{d}$ such that $m(t, x)=0$. The growth conditions (4) and (8) imply

$$
\begin{aligned}
C & \geq \int_{0}^{T} \int_{\mathbb{T}^{d}} F(x, m)+m H^{*}\left(x,-\frac{w}{m}\right) d t d x+\int_{\mathbb{T}^{d}} \phi_{T}(x) m(T, x) d x \\
& \geq \int_{0}^{T} \int_{\mathbb{T}^{d}}\left(\frac{1}{C}|m|^{q}+\frac{m}{C}\left|\frac{w}{m}\right|^{r^{\prime}}-C(m+1)\right) d x d t-\left\|\phi_{T}\right\|_{\infty} .
\end{aligned}
$$

Therefore $m \in L^{q}$. Moreover, by Hölder inequality, we also have

$$
\begin{aligned}
\int_{0}^{T} \int_{\mathbb{T}^{d}}|w|^{\frac{r^{\prime} q}{r^{\prime}+q-1}} & =\iint_{\{m>0\}}|w|^{\frac{r^{\prime} q}{r^{\prime}+q-1}} \\
& \leq\|m\|_{q}^{\frac{r^{\prime}-1}{r^{\prime}+q-1}}\left(\iint_{\{m>0\}} \frac{|w|^{r^{\prime}}}{m^{r^{\prime}-1}}\right)^{\frac{q}{r^{\prime}+q-1}} \leq C
\end{aligned}
$$

so that $w \in L^{\frac{r^{\prime} q}{r^{\prime}+q-1}}$. Finally, a minimizer to $(22)$ should be unique, because the set $\mathcal{K}_{1}$ is convex and the maps $F(x, \cdot)$ and $H^{*}(x, \cdot)$ are strictly convex: thus $m$ is unique and so is $\frac{w}{m}$ in $\{m>0\}$. As $w=0$ in $\{m=0\}$, uniqueness of $w$ follows as well. 


\section{Analysis of the optimal control of the HJ equation}

In general, we do not expect problem (17) to have a solution. In this section we exhibit a relaxation for (17) (Proposition 5.2) and show that this relaxed problem has at least one solution (Proposition 5.4).

\subsection{The relaxed problem}

Recall that the exponents $\eta>1$ and $\gamma>1$ are defined in Theorem 3.3. Let $\mathcal{K}$ be the set of pairs $(\phi, \alpha) \in L^{\gamma}\left((0, T) \times \mathbb{T}^{d}\right) \times L^{p}\left((0, T) \times \mathbb{T}^{d}\right)$ such that $D \phi \in L^{r}\left((0, T) \times \mathbb{T}^{d}\right)$ and which satisfy in the sense of distributions

$$
-\partial_{t} \phi-A_{i j}(x) \partial_{i j} \phi+H(x, D \phi) \leq \alpha, \quad \phi(T, \cdot) \leq \phi_{T}
$$

(for the precise meaning of the inequality, see the beginning of Sect. 3). The following statement explains that $\phi$ has a "trace" in a weak sense.

Lemma 5.1. Let $(\phi, \alpha) \in \mathcal{K}$. Then, for any Lipschitz continuous map $\zeta: \mathbb{T}^{d} \rightarrow$ $\mathbb{R}$, the map $t \rightarrow \int_{\mathbb{T}^{d}} \zeta(x) \phi(t, x) d x$ has a $B V$ representative on $[0, T]$. Moreover, if we denote by $\int_{\mathbb{T}^{d}} \zeta(x) \phi\left(t^{+}, x\right) d x$ its right limit at $t \in[0, T)$, then the map $\zeta \rightarrow \int_{\mathbb{T}^{d}} \zeta(x) \phi\left(t^{+}, x\right) d x$ is continuous in $L^{\eta^{\prime}}\left(\mathbb{T}^{d}\right)$.

As a consequence, for any nonnegative $C^{1} \operatorname{map} \vartheta:[0, T] \times \mathbb{T}^{d} \rightarrow \mathbb{R}$, one can write the integration by parts formula: for any $0 \leq t_{1} \leq t_{2} \leq T$,

$$
\begin{aligned}
& -\left[\int_{\mathbb{T}^{d}} \vartheta \phi\right]_{t_{1}}^{t_{2}}+\int_{t_{1}}^{t_{2}} \int_{\mathbb{T}^{d}} \phi \partial_{t} \vartheta+\langle D \vartheta, A D \phi\rangle+\vartheta\left(\partial_{i} A_{i j} \partial_{j} \phi+H(x, D \phi)\right) \\
& \quad \leq \int_{t_{1}}^{t_{2}} \int_{\mathbb{T}^{d}} \alpha \vartheta .
\end{aligned}
$$

Proof of Lemma 5.1. One easily checks that, for any Lipschitz continuous, nonnegative map $\zeta: \mathbb{T}^{d} \rightarrow \mathbb{R}$,

$$
-\frac{d}{d t} \int_{\mathbb{T}^{d}} \zeta \phi(t)+\int_{\mathbb{T}^{d}}\langle D \zeta, A D \phi(t)\rangle+\zeta\left(\partial_{i} A_{i j} \partial_{j} \phi+H(x, D \phi)-\alpha\right) \leq 0,
$$

holds in the sense of distributions. As the second integral is in $L^{1}((0, T))$, the map $t \rightarrow \int_{\mathbb{T}^{d}} \zeta \phi(t)$ is BV. If now $\zeta$ is Lipschitz continuous and changes sign, one can write $\zeta=\zeta^{+}-\zeta^{-}$and the map $t \rightarrow \int_{\mathbb{T}^{d}} \zeta \phi(t)=\int_{\mathbb{T}^{d}} \zeta^{+} \phi(t)-\int_{\mathbb{T}^{d}} \zeta^{-} \phi(t)$ is still BV. The continuity with respect to $\zeta$ comes from the $L^{\infty}\left((0, T), L^{\eta}\left(\mathbb{T}^{d}\right)\right)$ estimate on $\phi$ given in Theorem 3.3.

We extend the functional $\mathcal{A}$ to $\mathcal{K}$ by setting

$$
\mathcal{A}(\phi, \alpha)=\int_{0}^{T} \int_{\mathbb{T}^{d}} F^{*}(x, \alpha(t, x)) d x d t-\int_{\mathbb{T}^{d}} \phi(0, x) m_{0}(x) d x \quad \forall(\phi, \alpha) \in \mathcal{K} .
$$

The next proposition explains that the problem

$$
\inf _{(\phi, \alpha) \in \mathcal{K}} \mathcal{A}(\phi, \alpha)
$$


is the relaxed problem of (17). For this we first note that

$$
\inf _{(\phi, \alpha) \in \mathcal{K}} \mathcal{A}(\phi, \alpha)=\inf _{(\phi, \alpha) \in \mathcal{K}, \alpha \geq 0 \text { a.e. }} \mathcal{A}(\phi, \alpha)
$$

because one can always replace $\alpha$ by $\alpha \vee 0$ since $F^{*}(x, \alpha)=0$ for $\alpha \leq 0$.

Proposition 5.2. We have

$$
\inf _{\phi \in \mathcal{K}_{0}} \mathcal{A}(\phi)=\inf _{(\phi, \alpha) \in \mathcal{K}} \mathcal{A}(\phi, \alpha)
$$

The proof requires the following inequality:

Lemma 5.3. Let $(\phi, \alpha) \in \mathcal{K}$ and $(m, w) \in \mathcal{K}_{1}$. Assume that $m H^{*}(\cdot,-w / m) \in$ $L^{1}\left((0, T) \times \mathbb{T}^{d}\right)$ and $m \in L^{q}\left((0, T) \times \mathbb{T}^{d}\right)$. Then

$$
\left[\int_{\mathbb{T}^{d}} m \phi\right]_{t}^{T}+\int_{t}^{T} \int_{\mathbb{T}^{d}} m\left(\alpha+H^{*}\left(x,-\frac{w}{m}\right)\right) \geq 0
$$

and

$$
\left[\int_{\mathbb{T}^{d}} m \phi\right]_{0}^{t}+\int_{0}^{t} \int_{\mathbb{T}^{d}} m\left(\alpha+H^{*}\left(x,-\frac{w}{m}\right)\right) \geq 0 .
$$

Moreover, if equality holds in the inequality (26) for $t=0$, then $w=-m D_{p}$ $H(x, D \phi)$ a.e.

Proof. We first extend the pair $(m, w)$ to $[-1, T+1] \times \mathbb{T}^{d}$ by defining $m=m_{0}$ on $[-1,0], m=m(T)$ on $[T, T+1]$ and $w(s, x)=0$ for $(s, x) \in(-1,0) \cup(T, T+$ $1) \times \mathbb{T}^{d}$. Note that $\partial_{t} m-\partial_{i j}\left(\tilde{A}_{i j}(t, x) m\right)+\operatorname{div}(w)=0$ on $(-1, T+1) \times \mathbb{T}^{d}$, where $\tilde{A}_{i j}(t, x)=A_{i j}(x)$ if $t \in(0, T)$ and $\tilde{A}_{i j}(t, x)=0$ otherwise. Let $\xi^{\epsilon}=\xi^{\epsilon}(t, x)$ be a smooth convolution kernel with support in $B_{\epsilon}$; we smoothen the pair $(m, w)$ in a standard way into $\left(m_{\epsilon}, w_{\epsilon}\right)$. Then $\left(m_{\epsilon}, w_{\epsilon}\right)$ solves

$$
\partial_{t} m_{\epsilon}-\partial_{i j}\left(\tilde{A}_{i j} m_{\epsilon}\right)+\operatorname{div}\left(w_{\epsilon}\right)=\partial_{i} R_{\epsilon} \quad \text { in }(-1 / 2, T+1 / 2)
$$

in the sense of distributions, where

$$
R_{\epsilon}:=\left[\xi^{\epsilon}, \partial_{j} \tilde{A}_{i j}\right](m)+\left[\xi^{\epsilon}, \tilde{A}_{i j} \partial_{j}\right](m) .
$$

Here we use again the commutator notation (cf. [9])

$$
\left[\xi^{\epsilon}, c\right](f):=\xi^{\epsilon} \star(c f)-c\left(\xi^{\epsilon} \star f\right) .
$$

Invoking [9, Lemma II.1], we have that $R_{\epsilon} \rightarrow 0$ in $L^{q}$, since $m \in L^{q}$ and $\tilde{A}_{i j} \in W^{1, \infty}$.

Let us fix time $t \in(0, T)$ at which $\phi\left(t^{+}\right)=\phi\left(t^{-}\right)=\phi(t)$ in $L^{\gamma}(\mathbb{T})$ and $m_{\epsilon}(t)$ converges to $m(t)$. By the inequality satisfied by $(\phi, \alpha)$, we have

$$
\begin{aligned}
& \int_{t}^{T} \int_{\mathbb{T}^{d}} \phi \partial_{t} m_{\epsilon}+\partial_{i} \phi \partial_{j}\left(\tilde{A}_{i j} m_{\epsilon}\right)+m_{\epsilon} H(x, D \phi)+\int_{\mathbb{T}^{d}} m_{\epsilon}(t) \phi(t)-m_{\epsilon}(T) \phi_{T} \\
& \quad \leq \int_{t}^{T} \int_{\mathbb{T}^{d}} \alpha m_{\epsilon} .
\end{aligned}
$$

By (27) we have

$$
\int_{t}^{T} \int_{\mathbb{T}^{d}} \phi \partial_{t} m_{\epsilon}+\partial_{i} \phi \partial_{j}\left(\tilde{A}_{i j} m_{\epsilon}\right)=\int_{t}^{T} \int_{\mathbb{T}^{d}}-\partial_{i} \phi R_{\epsilon}+\left\langle D \phi, w_{\epsilon}\right\rangle .
$$


On the other hand, by convexity of $H$,

$$
\int_{t}^{T} \int_{\mathbb{T}^{d}}-m_{\epsilon} H^{*}\left(x,-\frac{w_{\epsilon}}{m_{\epsilon}}\right) \leq \int_{t}^{T} \int_{\mathbb{T}^{d}}\left\langle w_{\epsilon}, D \phi\right\rangle+m_{\epsilon} H(x, D \phi) .
$$

Collecting the above (in)equalities we obtain

$$
\int_{\mathbb{T}^{d}} m_{\epsilon}(t) \phi(t) \leq \int_{\mathbb{T}^{d}} m_{\epsilon}(T) \phi_{T}+\int_{t}^{T} \int_{\mathbb{T}^{d}} m_{\epsilon}\left(\alpha+H^{*}\left(x,-\frac{w_{\epsilon}}{m_{\epsilon}}\right)\right)+\partial_{j} \phi R_{\epsilon} .
$$

By assumption (7) which states that $r \geq p$, and since $D \phi \in L^{r}$, we have $\iint \partial_{j} \phi R_{\epsilon} \rightarrow 0$ as $\epsilon \rightarrow 0$. Following the proof of Lemma 2.7 in [4] we have

$$
\int_{t}^{T} \int_{\mathbb{T}^{d}}-m_{\epsilon} H^{*}\left(x,-\frac{w_{\epsilon}}{m_{\epsilon}}\right) \rightarrow \int_{t}^{T} \int_{\mathbb{T}^{d}}-m H^{*}\left(x,-\frac{w}{m}\right) \quad \text { as } \epsilon \rightarrow 0 .
$$

The continuity of $t \rightarrow m(t)$ in $P\left(\mathbb{T}^{d}\right)$ given by Lemma 4.1 implies the convergence

$$
\int_{\mathbb{T}^{d}} m_{\epsilon}(T) \phi_{T} \rightarrow \int_{\mathbb{T}^{d}} m(T) \phi_{T}
$$

Recalling that $\phi \in L^{\gamma}\left((0, T) \times \mathbb{T}^{d}\right), m \in L^{q}\left((0, T) \times \mathbb{T}^{d}\right)$ and $\gamma \geq p, m_{\epsilon} \phi$ strongly converges to $m \phi$ in $L^{1}\left((0, T) \times \mathbb{T}^{d}\right)$ and, therefore, up to a subsequence, $\int_{\mathbb{T}^{d}} m_{\epsilon}(t) \phi(t) \rightarrow \int_{\mathbb{T}^{d}} m(t) \phi(t)$ a.e. Hence

$$
\int_{\mathbb{T}^{d}} m(t) \phi(t) \leq \int_{\mathbb{T}^{d}} m(T) \phi_{T}+\int_{t}^{T} \int_{\mathbb{T}^{d}} m\left(\alpha+H^{*}\left(x,-\frac{w}{m}\right)\right) .
$$

We can argue similarly in the time interval $[0, t]$ and obtain

$$
\int_{\mathbb{T}^{d}} m_{0} \phi(0) \leq \int_{\mathbb{T}^{d}} m(t) \phi(t)+\int_{0}^{t} \int_{\mathbb{T}^{d}} m\left(\alpha+H^{*}\left(x,-\frac{w}{m}\right)\right) .
$$

Let us assume finally that the following equality holds:

$$
\left[\int_{\mathbb{T}^{d}} m \phi\right]_{0}^{T}+\int_{0}^{T} \int_{\mathbb{T}^{d}} m\left(\alpha+H^{*}\left(x,-\frac{w}{m}\right)\right)=0
$$

Then there is an equality in inequality (26) for almost all $t$. Fix such a $t \in(0, T)$ and let

$E_{\sigma}(t):=\left\{(s, y), s \in[t, T], m\left(H^{*}\left(y,-\frac{w}{m}\right)+H(x, D \phi)\right) \geq-\langle w, D \phi\rangle+\sigma\right\}$. 
If $\left|E_{\sigma}(t)\right|>0$, then for $\epsilon>0$ small enough, the set

$E_{\epsilon, \sigma}(t):=\left\{(s, y), s \in[t, T], m_{\epsilon}\left(H^{*}\left(y,-\frac{w_{\epsilon}}{m_{\epsilon}}\right)+H(x, D \phi)\right) \geq-\left\langle w_{\epsilon}, D \phi\right\rangle+\sigma / 2\right\}$

has a measure larger than $\left|E_{\sigma}(t)\right| / 2$. Coming back to inequality (30), we have

$$
\int_{t}^{T} \int_{\mathbb{T}^{d}}-m_{\epsilon} H^{*}\left(x,-\frac{w_{\epsilon}}{m_{\epsilon}}\right) \leq \int_{t}^{T} \int_{\mathbb{T}^{d}}\left\langle w_{\epsilon}, D \phi\right\rangle+m_{\epsilon} H(x, D \phi)-\left|E_{\sigma}(t)\right| \sigma / 4
$$

Then inequality (26) becomes

$$
\int_{\mathbb{T}^{d}} m(t) \phi(t) \leq \int_{\mathbb{T}^{d}} m(T) \phi_{T}+\int_{t}^{T} \int_{\mathbb{T}^{d}} m\left(\alpha+H^{*}\left(x,-\frac{w}{m}\right)\right)-\left|E_{\sigma}(t)\right| \sigma / 4,
$$

which contradicts the fact that there is an equality in (26). So $\left|E_{\sigma}(t)\right|=0$ for any $\sigma$ and for a.e. $t$, which shows that $m\left(H^{*}\left(y,-\frac{w}{m}\right)+H(x, D \phi)\right)=-\langle w, D \phi\rangle$ a.e. Thus $w=-m D_{p} H(x, D \phi)$ holds a.e. in $\{m>0\}$ and, as $w=0$ in $\{m=0\}$, a.e. in $(0, T) \times \mathbb{T}^{d}$.

Proof of Proposition 5.2. We follow the argument developed by Graber in [14]. Inequality $\inf _{\phi \in \mathcal{K}_{0}} \mathcal{A}(\phi) \geq \inf _{(\phi, \alpha) \in \mathcal{K}} \mathcal{A}(\phi, \alpha)$ being obvious, let us check the reverse one. Let $(\phi, \alpha) \in \mathcal{K}$. For any $(m, w) \in \mathcal{K}_{1}$ with $m H^{*}\left(\cdot,-\frac{w}{m}\right) \in L^{1}$, we have, by Lemma 5.3,

$$
\begin{aligned}
\mathcal{A}(\phi, \alpha) & \geq \int_{0}^{T} \int_{\mathbb{T}^{d}} \alpha m-F(m)-\int_{\mathbb{T}^{d}} m_{0} \phi(0) \\
& \geq \int_{0}^{T} \int_{\mathbb{T}^{d}}-m H^{*}\left(x,-\frac{w}{m}\right)-F(m)-\int_{\mathbb{T}^{d}} m(T) \phi_{T}=-\mathcal{B}(m, w)
\end{aligned}
$$

Taking the sup with respect to $(m, w)$ in the right-hand side we obtain thanks to Lemma 4.2:

$$
\mathcal{A}(\phi, \alpha) \geq-\inf _{(m, w) \in \mathcal{K}_{1}} \mathcal{B}(m, w)=\inf _{\phi \in \mathcal{K}_{0}} \mathcal{A}(\phi) .
$$

\subsection{Existence of a solution for the relaxed problem}

The next proposition explains the interest of considering the relaxed problem (24) instead of the original one (17).

Proposition 5.4. The relaxed problem (24) has at least one solution $(\phi, \alpha) \in \mathcal{K}$ which is bounded below by a constant depending on $\left\|\phi_{T}\right\|_{C^{2}}$, on $\left\|A_{i j}\right\|_{C^{0}}$ and on $\left\|H\left(\cdot, D \phi_{T}\right)\right\|_{\infty}$.

Proof. We start with the construction of a suitable minimizing sequence. Let $\left(\tilde{\phi}_{n}\right)$ be a minimizing sequence for problem (17) and let us set

$$
\alpha_{n}(t, x)=\max \left\{0 ;-\partial_{t} \tilde{\phi}_{n}(t, x)-A_{i j} \partial_{i j} \tilde{\phi}_{n}(t, x)+H\left(x, D \tilde{\phi}_{n}(t, x)\right)\right\} .
$$

By Proposition 5.2 and the fact that $F^{*}(x, \alpha)=0$ if $\alpha \leq 0$, the pair $\left(\tilde{\phi}_{n}, \alpha_{n}\right)$ is also a minimizing sequence of $(24)$. Let $\psi$ be the unique viscosity solution to

$$
-\partial_{t} \psi-A_{i j}(x) \partial_{i j} \psi+H(x, D \psi)=0, \quad \psi(T, \cdot)=\phi_{T}
$$


As $\phi_{T}$ is $C^{2}, \psi(t, x) \geq \phi_{T}(x)-C(T-t)$, where the constant $C$ depends on $\left\|\phi_{T}\right\|_{C^{2}}$, on $\left\|A_{i j}\right\|_{C^{0}}$ and on $\left\|H\left(\cdot, D \phi_{T}\right)\right\|_{\infty}$. Let $\phi_{n}$ be the (continuous) viscosity solution to

$$
-\partial_{t} \phi_{n}-A_{i j}(x) \partial_{i j} \phi_{n}+H\left(x, D \phi_{n}\right)=\alpha_{n}, \quad \phi_{n}(T, \cdot)=\phi_{T} .
$$

By comparison, $\phi_{n} \geq \tilde{\phi}_{n} \vee \psi$. As $H$ is convex, (32) holds in the sense of distributions (see [16]). Therefore the sequence $\left(\phi_{n}, \alpha_{n}\right)$ is still minimizing, with the following bound below for $\left(\phi_{n}\right)$ :

$$
\phi_{n}(t, x) \geq \phi_{T}(x)-C(T-t) .
$$

Step 1 We claim that $\left(\alpha_{n}\right)$ is bounded in $L^{p}\left((0, T) \times \mathbb{T}^{d}\right)$. For this, we integrate (32) against $m_{0}$ on $(0, T) \times \mathbb{T}^{d}$

$$
\begin{aligned}
& \int_{\mathbb{T}^{d}} \phi_{n}(0) m_{0}+\int_{y} 0^{T} \int_{\mathbb{T}^{d}} \partial_{i} m_{0} A_{i j} \partial_{j} \phi_{n}+\left(\partial_{j} A_{i j}\right) m_{0} \partial_{j} \phi_{n}+m_{0} H\left(x, D \phi_{n}\right) \\
& \leq \int_{0}^{T} \int_{\mathbb{T}^{d}} m_{0} \alpha_{n}+\int_{\mathbb{T}^{d}} \phi_{T} m_{0} .
\end{aligned}
$$

As $\left(1 / C_{0}\right) \leq m_{0} \leq C_{0}$ for some $C_{0}>0,\left\|D m_{0}\right\|_{\infty}<+\infty$ and $H$ is coercive, we get

$$
\int_{\mathbb{T}^{d}} \phi_{n}(0) m_{0}+\frac{1}{C} \int_{0}^{T} \int_{\mathbb{T}^{d}}\left|D \phi_{n}\right|^{r} \leq C_{0}\left\|\alpha_{n}\right\|_{p}+C .
$$

On the other hand, as $\left(\phi_{n}\right)$ is a minimizing sequence and $F^{*}$ is coercive,

$$
\frac{1}{C}\left\|\alpha_{n}\right\|_{p}^{p}-\int_{\mathbb{T}^{d}} \phi_{n}(0) m_{0} \leq \int_{0}^{T} \int_{\mathbb{T}^{d}} F^{*}\left(x, \alpha_{n}\right)-\int_{\mathbb{T}^{d}} \phi_{n}(0) m_{0}+C \leq C .
$$

Adding the previous inequalities, we get

$$
\frac{1}{C}\left\|\alpha_{n}\right\|_{p}^{p}+\frac{1}{C} \int_{0}^{T} \int_{\mathbb{T}^{d}}\left|D \phi_{n}\right|^{r} \leq C_{0}\left\|\alpha_{n}\right\|_{p}+C,
$$

so that $\left(\alpha_{n}\right)$ is bounded in $L^{p}\left((0, T) \times \mathbb{T}^{d}\right)$ while $\left(D \phi_{n}\right)$ is bounded in $L^{r}$.

Step 2 We show here that $\left(\phi_{n}, \alpha_{n}\right)$ has a limit. As $\left(\alpha_{n}\right)$ is bounded in $L^{p}$ and $\left(\phi_{n}\right)$ is uniformly bounded below thanks to (33), Theorem 3.3 implies that $\left(\phi_{n}\right)$ is bounded in $L^{\gamma}$. So we can assume with loss of generality that $\alpha_{n} \rightarrow \bar{\alpha}$ in $L^{p}, \phi_{n} \rightarrow \bar{\phi}$ in $L^{\gamma}$ and $D \phi_{n} \rightarrow D \bar{\phi}$ in $L^{r}$ where, in view of the convexity of $H$, the pair $(\bar{\phi}, \bar{\alpha})$ belongs to $\mathcal{K}$.

Step 3 We now prove that $(\bar{\phi}, \bar{\alpha})$ is a minimizer. By weak lower semicontinuity arguments, we have

$$
\liminf _{n} \int_{0}^{T} \int_{\mathbb{T}^{d}} F^{*}\left(x, \alpha_{n}\right) \geq \int_{0}^{T} \int_{\mathbb{T}^{d}} F^{*}(x, \bar{\alpha}) .
$$

Let $\zeta_{n}(t)=\int_{\mathbb{T}^{d}} m_{0} \phi_{n}(t)$ and $\bar{\zeta}(t)=\int_{\mathbb{T}^{d}} m_{0} \bar{\phi}(t)$. Then $\left(\zeta_{n}\right)$ converges weak ${ }^{*}$ to $\bar{\zeta}$ in $L^{\infty}$ thanks to Theorem 3.3. As

$$
-\frac{d}{d t} \zeta_{n}(t)+\int_{\mathbb{T}^{d}}\left\langle D m_{0}, A D \phi_{n}(t)\right\rangle+m_{0}\left(\partial_{i} A_{i j} \partial_{j} \phi_{n}+H\left(x, D \phi_{n}\right)-\alpha_{n}\right) \leq 0
$$


we also have by coercivity of $H$ and thanks to the bound on $\left(\alpha_{n}\right)$ :

$$
\zeta_{n}(0)-C t^{\frac{1}{p^{\prime}}} \leq \zeta_{n}(t) \quad \forall t \in[0, T]
$$

Letting $n \rightarrow+\infty$ :

$$
\limsup _{n} \zeta_{n}(0)-C t^{\frac{1}{p^{\prime}}} \leq \bar{\zeta}(t) \quad \text { a.e. } t \in[0, T]
$$

so that $\lim \sup _{n} \zeta_{n}(0) \leq \int_{\mathbb{T}^{d}} m_{0} \bar{\phi}(0)$. Hence

$$
\liminf _{n} \int_{0}^{T} \int_{\mathbb{T}^{d}} F^{*}\left(x, \alpha_{n}\right)-\int_{\mathbb{T}^{d}} m_{0} \phi_{n}(0) \geq \int_{0}^{T} \int_{\mathbb{T}^{d}} F^{*}(x, \bar{\alpha})-\int_{\mathbb{T}^{d}} m_{0} \bar{\phi}(0)
$$

and $(\bar{\phi}, \bar{\alpha})$ is a minimum.

Remark 5.5. If $r>2$ and $p>1+d / r$, then by [6] the sequence $\left(\phi_{n}\right)$ built at the beginning of the proof is uniformly Hölder continuous. Hence so is $\phi$.

\section{Existence and uniqueness of a solution for the MFG system}

In this section we show that the MFG system (1) has a unique weak solution and prove the stability of this solution with respect to the data.

\subsection{Definition of weak solutions}

The variational method described above provides weak solutions for the MFG system. By a weak solution, we mean the following:

Definition 6.1. We say that a pair $(\phi, m) \in L^{\gamma}\left((0, T) \times \mathbb{T}^{d}\right) \times L^{q}\left((0, T) \times \mathbb{T}^{d}\right)$ is a weak solution to (1) if

(i) the following integrability conditions hold:

$D \phi \in L^{r}, m H^{*}\left(\cdot, D_{p} H(\cdot, D \phi)\right) \in L^{1} \quad$ and $\left.\quad m D_{p} H(\cdot, D \phi)\right) \in L^{1}$.

(ii) Equation (1)-(i) holds in the following sense: inequality

$$
-\partial_{t} \phi-\partial_{i}\left(A_{i j}(x) \partial_{j} \phi\right)+\left(\partial_{i} A_{i j}\right) \partial_{j} \phi+H(x, D \phi) \leq f(x, m) \quad \text { in }(0, T) \times \mathbb{T}^{d},
$$

with $\phi(T, \cdot) \leq \phi_{T}$, holds in the sense of distributions,

(iii) Equation (1)-(ii) holds:

$\left.\partial_{t} m-\partial_{i j}\left(A_{i j}(x) m\right)-\operatorname{div}\left(m D_{p} H(x, D \phi)\right)\right)=0$ in $(0, T) \times \mathbb{T}^{d}, \quad m(0)=m_{0}$

in the sense of distributions,

(iv) The following equality holds:

$$
\begin{aligned}
& \int_{0}^{T} \int_{\mathbb{T}^{d}} m(t, x)\left(f(x, m(t, x))+H^{*}\left(x, D_{p} H(x, D \phi)(t, x)\right)\right) d x d t \\
& \quad+\int_{\mathbb{T}^{d}} m(T, x) \phi_{T}(x)-m_{0}(x) \phi(0, x) d x=0 .
\end{aligned}
$$

Notice that last term in (37) is well defined due to Lemma 5.1. 
Our main result is the following existence and uniqueness theorem:

Theorem 6.2. There exists a weak solution $(\phi, m)$ to the MFG system (1). Moreover this solution is unique in the following sense: if $(\phi, m)$ and $\left(\phi^{\prime}, m^{\prime}\right)$ are two solutions, then $m=m^{\prime}$ a.e. and $\phi=\phi^{\prime}$ in $\{m>0\}$.

Finally, there exists a solution which is bounded below by a constant depending on $\left\|\phi_{T}\right\|_{C^{2}}$, on $\left\|A_{i j}\right\|_{C^{0}}$ and on $\left\|H\left(\cdot, D \phi_{T}\right)\right\|_{\infty}$.

Remark 6.3. Under the assumptions of Remark 5.5, i.e., if $r>2$ and $p>$ $1+d / r$, the $\phi$-component of the solution is locally Hölder continuous.

\subsection{Existence of a weak solution}

The first step towards the proof of Theorem 6.2 consists in showing a one-toone equivalence between solutions of the MFG system and the two optimization problems (22) and (24).

Theorem 6.4. Let $(\bar{m}, \bar{w}) \in \mathcal{K}_{1}$ be a minimizer of $(22)$ and $(\bar{\phi}, \bar{\alpha}) \in \mathcal{K}$ be a minimizer of (24). Then $(\bar{\phi}, \bar{m})$ is a weak solution of the mean field games system (1) and $\bar{w}=-\bar{m} D_{p} H(\cdot, D \bar{\phi})$ while $\bar{\alpha}=f(\cdot, \bar{m})$ a.e..

Conversely, any weak solution $(\bar{\phi}, \bar{m})$ of $(1)$ is such that the pair $\left(\bar{m},-\bar{m} D_{p} H\right.$ $(\cdot, D \bar{\phi}))$ is the minimizer of $(22)$ while $(\bar{\phi}, f(\cdot, \bar{m}))$ is a minimizer of $(24)$.

Proof. Let $(\bar{m}, \bar{w}) \in \mathcal{K}_{1}$ be a minimizer of Problem $(22)$ and $(\bar{\phi}, \bar{\alpha}) \in \mathcal{K}$ be a minimizer of Problem (24). Due to Lemma 4.2 and Proposition 5.2, we have

$$
\begin{aligned}
& \int_{0}^{T} \int_{\mathbb{T}^{d}} F^{*}(x, \bar{\alpha})+F(x, \bar{m})+\bar{m} H^{*}\left(x,-\frac{\bar{w}}{\bar{m}}\right) d x d t \\
& \quad+\int_{\mathbb{T}^{d}} \phi_{T} \bar{m}(T)-\bar{\phi}(0) m_{0} d x=0 .
\end{aligned}
$$

We show that $\bar{\alpha}=f(x, \bar{m})$. Indeed, by convexity of $F$,

$$
F^{*}(x, \bar{\alpha}(t, x))+F(x, \bar{m}(t, x))-\bar{\alpha}(t, x) \bar{m}(t, x) \geq 0,
$$

hence

$$
\int_{0}^{T} \int_{\mathbb{T}^{d}} \bar{\alpha}(t, x) \bar{m}(t, x)+\bar{m} H^{*}\left(x,-\frac{\bar{w}}{\bar{m}}\right) d x d t+\int_{\mathbb{T}^{d}} \phi_{T} \bar{m}(T)-\bar{\phi}(0) m_{0} d x \leq 0 .
$$

Thanks to Lemma 5.3, the above inequality is in fact an equality, $\bar{w}=-\bar{m} D_{p} H(\cdot, D \bar{\phi})$ a.e. and the equality holds almost everywhere in Eq. (38). Therefore,

$$
\bar{\alpha}(t, x)=f(x, \bar{m}(t, x))
$$

almost everywhere and (37) holds:

$$
\int_{0}^{T} \int_{\mathbb{T}^{d}} f \bar{m}+\bar{m} H^{*}\left(x,-\frac{\bar{w}}{\bar{m}}\right) d x d t+\int_{\mathbb{T}^{d}} \phi_{T} \bar{m}(T)-\bar{\phi}(0) m_{0} d x=0 .
$$

In particular $\bar{m} H^{*}\left(\cdot, D_{\underline{p}} H(\cdot, D \bar{\phi})\right) \in L^{1}$.

Moreover, since $(\bar{\phi}, \bar{\alpha}) \in \mathcal{K}$ and Eq. (39) holds, we have $-\partial_{t} \bar{\phi}-A_{i j} \partial_{i j} \bar{\phi}+$ $H(x, D \bar{\phi}) \leq f(x, \bar{m})$ in the sense of distributions and $\bar{\phi}(T) \leq \phi_{T}$.

Furthermore, since $(\bar{\phi}, \bar{\alpha}) \in \mathcal{K}$ and $\bar{w}=-\bar{m} D_{p} H(\cdot, D \bar{\phi})$, we have that $\bar{m} D_{p} H(\cdot, D \bar{\phi}) \in L^{1}$ and (36) holds in the sense of distributions. 
Therefore $(\bar{\phi}, \bar{m})$ is a solution in the sense of Definition 6.1 .

Suppose now that $(\bar{\phi}, \bar{m})$ is a weak solution of (1) as in Definition 6.1. Set $\bar{w}=-\bar{m} D_{p} H(\cdot, D \bar{\phi})$ and $\bar{\alpha}(t, x)=f(x, \bar{m}(t, x))$. By definition of weak solution $\bar{w}, \bar{\alpha} \in L^{1}, \bar{m} \in L^{q}$ and $\bar{\phi} \in L^{\gamma}$. Moreover, since $\bar{m} \in L^{q}$, the growth condition (8) implies that $\bar{\alpha} \in L^{p}$. Therefore $(\bar{m}, \bar{w}) \in \mathcal{K}_{1}$ and $(\bar{\phi}, \bar{\alpha}) \in \mathcal{K}$.

It remains to show that $(\bar{\phi}, \bar{\alpha})$ minimizes $\mathcal{A}$ and $(\bar{m}, \bar{w})$ minimizes $\mathcal{B}$.

Let $\left(\bar{\phi}^{\prime}, \bar{\alpha}^{\prime}\right) \in \mathcal{K}$. By the convexity of $F$ in the second variable, we have

$$
\begin{aligned}
\mathcal{A}\left(\bar{\phi}^{\prime}, \bar{\alpha}^{\prime}\right)= & \int_{0}^{T} \int_{\mathbb{T}^{d}} F^{*}\left(x, \bar{\alpha}^{\prime}(t, x)\right) d x d t-\int_{\mathbb{T}^{d}} \bar{\phi}^{\prime}(0, x) m_{0}(x) d x \\
\geq & \int_{0}^{T} \int_{\mathbb{T}^{d}} F^{*}(x, \bar{\alpha}(t, x))+\partial_{\alpha} F^{*}(x, \bar{\alpha}(t, x))\left(\bar{\alpha}^{\prime}(t, x)-\bar{\alpha}(t, x)\right) d x d t \\
& -\int_{\mathbb{T}^{d}} \bar{\phi}^{\prime}(0, x) m_{0}(x) d x \\
\geq & \int_{0}^{T} \int_{\mathbb{T}^{d}} F^{*}(x, \bar{\alpha}(t, x))+\bar{m}(t, x)\left(\bar{\alpha}^{\prime}(t, x)-\bar{\alpha}(t, x)\right) d x d t \\
& -\int_{\mathbb{T}^{d}} \bar{\phi}^{\prime}(0, x) m_{0}(x) d x, \\
\geq & \mathcal{A}(\bar{\phi}, \bar{\alpha})+\int_{0}^{T} \int_{\mathbb{T}^{d}} \bar{m}(t, x)\left(\bar{\alpha}^{\prime}(t, x)-\bar{\alpha}(t, x)\right) d x d t \\
& +\int_{\mathbb{T}^{d}}\left(\bar{\phi}(0, x)-\bar{\phi}^{\prime}(0, x)\right) m_{0}(x) d x .
\end{aligned}
$$

Due to Eq. (37) and Lemma 5.3 applied to $\left(\bar{\phi}^{\prime}, \bar{\alpha}^{\prime}\right)$ and $(\bar{m}, \bar{w})$ we have

$$
\begin{aligned}
\int_{0}^{T} & \int_{\mathbb{T}^{d}} \bar{m}(t, x)\left(\bar{\alpha}^{\prime}(t, x)-\bar{\alpha}(t, x)\right) d x d t+\int_{\mathbb{T}^{d}}\left(\bar{\phi}(0, x)-\bar{\phi}^{\prime}(0, x)\right) m_{0}(x) d x \\
= & \int_{0}^{T} \int_{\mathbb{T}^{d}} \bar{m}(t, x) \bar{\alpha}^{\prime}(t, x)+\bar{m}(t, x) H^{*}\left(x,-\frac{\bar{w}(t, x)}{\bar{m}(t, x)}\right) d x d t \\
& +\int_{\mathbb{T}^{d}} \phi_{T}(x) \bar{m}(T, x)-\bar{\phi}^{\prime}(0, x) m_{0}(x) d x \geq 0 .
\end{aligned}
$$

Hence,

$$
\mathcal{A}\left(\bar{\phi}^{\prime}, \bar{\alpha}^{\prime}\right) \geq \mathcal{A}(\bar{\phi}, \bar{\alpha})
$$

and $(\bar{\phi}, \bar{\alpha})$ is a minimizer of $\mathcal{A}$.

The argument for $(\bar{m}, \bar{w})$ is similar. Let $\left(\bar{m}^{\prime}, \bar{w}^{\prime}\right)$ minimize $\mathcal{B}$. Then because $F$ is convex in the second variable, we have

$$
\begin{aligned}
\mathcal{B}\left(\bar{m}^{\prime}, \bar{w}^{\prime}\right) & =\int_{\mathbb{T}^{d}} \phi_{T} \bar{m}^{\prime}(T)+\iint \bar{m}^{\prime} H^{*}\left(x,-\frac{\bar{w}^{\prime}}{\bar{m}^{\prime}}\right)+F\left(x, \bar{m}^{\prime}\right) \\
& \geq \int_{\mathbb{T}^{d}} \phi_{T} \bar{m}^{\prime}(T)+\iint \bar{m}^{\prime} H^{*}\left(x,-\frac{\bar{w}^{\prime}}{\bar{m}^{\prime}}\right)+F(x, \bar{m})+f(x, \bar{m})\left(\bar{m}^{\prime}-\bar{m}\right) \\
& =\int_{\mathbb{T}^{d}} \phi_{T} \bar{m}^{\prime}(T)+\iint \bar{m}^{\prime} H^{*}\left(x,-\frac{\bar{w}^{\prime}}{\bar{m}^{\prime}}\right)+F(x, \bar{m})+\bar{\alpha}\left(\bar{m}^{\prime}-\bar{m}\right)
\end{aligned}
$$




$$
\begin{aligned}
& =\mathcal{B}(\bar{m}, \bar{w})+\int_{\mathbb{T}^{d}} \phi_{T} \bar{m}^{\prime}(T)-m_{0} \bar{\phi}(0)+\iint \bar{m}^{\prime} H^{*}\left(x,-\frac{\bar{w}^{\prime}}{\bar{m}^{\prime}}\right)+\bar{\alpha} \bar{m}^{\prime} \\
& \geq \mathcal{B}(\bar{m}, \bar{w}) .
\end{aligned}
$$

Here we used Eq. (37) in the next to last line, and we applied Lemma 5.3 to $(\bar{\phi}, \bar{\alpha})$ and $\left(\bar{m}^{\prime}, \bar{w}^{\prime}\right)$ in the last line. Therefore $(\bar{m}, \bar{w})$ is a minimizer of $\mathcal{B}$.

\subsection{Uniqueness of the weak solution}

Proof of Theorem 6.2 (uniqueness part). Let $(\bar{\phi}, \bar{m})$ be a weak solution to (1). In view of Theorem 6.4 , the pair $\left(\bar{m},-\bar{m} D_{p} H(\cdot, D \bar{\phi})\right)$ is the minimizer of $(22)$ while $(\bar{\phi}, f(\cdot, \bar{m}))$ is a solution of $(24)$. In particular, $\bar{m}$ is unique because of the uniqueness of the solution of $(22)$.

Let now $\left(\phi_{1}, \bar{m}\right)$ and $\left(\phi_{2}, \bar{m}\right)$ be two weak solutions of $(1)$, and set $\bar{\alpha}=$ $f(\cdot, \bar{m})$. Let $\bar{\phi}=\phi_{1} \vee \phi_{2}$. Assume for now that $\bar{\phi}$ is a subsolution of (23) in the sense of distributions. Then $(\bar{\phi}, \bar{\alpha}) \in \mathcal{K}$, and so because $-\int \bar{\phi}(0) m_{0} \leq$ $-\int \phi_{1}(0) m_{0}$ we have that $(\bar{\phi}, \bar{\alpha})$ is also a solution of $(24)$. Indeed, one deduces from Lemma 5.3 that for a.e. $t \in[0, T],(\bar{\phi}, \bar{\alpha})$ and $\left(\phi_{1}, \bar{\alpha}\right)$, are both minimizers of the problem

$$
\inf _{(\phi, \alpha) \in \mathcal{K}} \int_{t}^{T} \int_{\mathbb{T}^{d}} F^{*}(x, \alpha)-\int_{\mathbb{T}^{d}} \bar{m}(t) \phi(t) .
$$

In particular, $\int_{\mathbb{T}^{d}} \bar{m}(t) \bar{\phi}(t)=\int_{\mathbb{T}^{d}} \bar{m}(t) \phi_{1}(t)$. As $\phi_{1} \leq \bar{\phi}$, this implies that $\phi_{1}=\bar{\phi}$ a.e. in $\{\bar{m}>0\}$. The same argument, replacing $\phi_{1}$ with $\phi_{2}$, shows that $\phi_{2}=\bar{\phi}$ a.e. in $\{\bar{m}>0\}$, and uniqueness is proved.

The main difficulty is to show that $\bar{\phi}=\phi_{1} \vee \phi_{2}$ is indeed a subsolution of $(23)$ in the sense of distributions, i.e.

$$
\begin{aligned}
& -\int_{\mathbb{T}^{d}} \zeta(T) \phi_{T}+\int_{0}^{T} \int_{\mathbb{T}^{d}} \bar{\phi} \partial_{t} \zeta+\langle D \zeta, A D \bar{\phi}\rangle+\zeta\left(\partial_{i} A_{i j} \partial_{j} \bar{\phi}+H(x, D \bar{\phi})\right) \\
& \quad \leq \int_{0}^{T} \int_{\mathbb{T}^{d}} \bar{\alpha} \zeta
\end{aligned}
$$

for any nonnegative smooth map $\zeta$ with support in $(0, T] \times \mathbb{T}^{d}$.

Let $\epsilon>0$. Introduce the following translation and extension of $\left(\phi_{k}, \bar{\alpha}\right)$, $k=1,2$ :

$$
\tilde{\phi}_{k}(t, x)= \begin{cases}\phi_{k}(t+2 \epsilon, x) & \text { if } t \in[-2 \epsilon, T-2 \epsilon) \\ \phi_{T}(x) & \text { if } t \in[T-2 \epsilon, T+2 \epsilon]\end{cases}
$$

and

$$
\tilde{\alpha}(t, x)= \begin{cases}\bar{\alpha}(t+2 \epsilon, x) & \text { if } t \in[-2 \epsilon, T-2 \epsilon) \\ \lambda & \text { if } t \in[T-2 \epsilon, T+2 \epsilon]\end{cases}
$$

where $\lambda=\max _{x} H\left(x, D \phi_{T}(x)\right)+A_{i j}(x) \partial_{i j} \phi_{T}(x)$. Then we have that

$$
-\partial_{t} \tilde{\phi}_{k}-A_{i j} \partial_{i j} \tilde{\phi}_{k}+H\left(x, D \tilde{\phi}_{k}\right) \leq \tilde{\alpha}
$$

in the sense of distributions on $(-2 \epsilon, T+2 \epsilon) \times \mathbb{T}^{d}$. 
For now we will fix a smooth vector field $\psi$ on $[0, T] \times \mathbb{T}^{d}$. Notice that

$$
-\partial_{t} \tilde{\phi}_{k}-A_{i j} \partial_{i j} \tilde{\phi}_{k}+\psi \cdot D \tilde{\phi}_{k} \leq \tilde{\alpha}+H^{*}(x, \psi)
$$

in the sense of distributions on $(-2 \epsilon, T+2 \epsilon) \times \mathbb{T}^{d}$.

Let $\xi^{1}$ be a smooth convolution kernel in $\mathbb{R}^{d+1}$ with support in the unit ball, with $\xi^{1} \geq 0$ and $\int \xi^{1}=1$. Then define the standard mollifier sequence $\xi^{\epsilon}(t, x)=\epsilon^{-d-1} \xi^{1}((t, x) / \epsilon)$. Set $\phi_{k}^{\epsilon}=\xi^{\epsilon} \star \tilde{\phi}_{k}$ and $\alpha^{\epsilon}=\xi^{\epsilon} \star \tilde{\alpha}$. By taking the convolution we have, in a pointwise sense,

$$
-\partial_{t} \phi_{k}^{\epsilon}-A_{i j} \partial_{i j} \phi_{k}^{\epsilon}+\psi \cdot D \phi_{k}^{\epsilon} \leq \alpha^{\epsilon}+\xi^{\epsilon} \star H^{*}(\cdot, \psi)+R_{\epsilon}^{k}-S_{\epsilon}^{k}
$$

on $[0, T] \times \mathbb{T}^{d}$, where

$$
R_{\epsilon}^{k}:=\left[\xi^{\epsilon}, A_{i j} \partial_{j}\right]\left(\partial_{i} \tilde{\phi}_{k}\right), \quad S_{\epsilon}^{k}:=\left[\xi^{\epsilon}, \psi\right]\left(D \tilde{\phi}_{k}\right) .
$$

Here we use the same commutator notation as in (29). Invoking [9, Lemma II.1], we have that $R_{\epsilon}^{k}$ and $S_{\epsilon}^{k}, k=1,2$ are smooth functions which converge to zero in $L^{r}$, since $A_{i j} \in W^{1, \infty}$ is given and $\psi$ may also be chosen in $W^{1, \infty}$.

Define $R_{\epsilon}:=\max \left\{R_{\epsilon}^{1}-S_{\epsilon}^{1}, R_{\epsilon}^{2}-S_{\epsilon}^{2}\right\}$. This, too, converges to zero in $L^{r}$. Moreover, for $k=1,2$

$$
-\partial_{t} \phi_{k}^{\epsilon}-A_{i j} \partial_{i j} \phi_{k}^{\epsilon}+\psi \cdot D \phi_{k}^{\epsilon} \leq \alpha^{\epsilon}+\xi^{\epsilon} \star H^{*}(\cdot, \psi)+R_{\epsilon}
$$

holds in a pointwise sense, hence also in a viscosity sense. By standard results, (47) holds also for $\phi^{\epsilon}:=\phi_{1}^{\epsilon} \vee \phi_{2}^{\epsilon}$ in a viscosity sense. The result of [16] implies that it also holds in the sense of distributions, that is, for any smooth map $\zeta$ with support in $(0, T] \times \mathbb{T}^{d}$ we have

$$
\begin{aligned}
& -\int_{\mathbb{T}^{d}} \zeta(T) \phi^{\epsilon}(T)+\int_{0}^{T} \int_{\mathbb{T}^{d}} \phi^{\epsilon} \partial_{t} \zeta+\left\langle D \zeta, A D \phi^{\epsilon}\right\rangle+\zeta\left(\partial_{i} A_{i j} \partial_{j} \phi^{\epsilon}+D \phi^{\epsilon} \cdot \psi\right) \\
& \leq \int_{0}^{T} \int_{\mathbb{T}^{d}} \zeta\left(\alpha^{\epsilon}+\xi^{\epsilon} \star H^{*}(\cdot, \psi)+R_{\epsilon}\right) .
\end{aligned}
$$

By construction, $\phi^{\epsilon}(T)=\phi_{T}$ for all $\epsilon>0$. Observe that $\phi^{\epsilon} \rightarrow \bar{\phi}$ in $L^{\gamma}$ and $D \phi^{\epsilon} \rightarrow D \bar{\phi}$ in $L^{r}$, as these sequences are only slight adaptations of classical convolutions of $\bar{\phi}$ and $D \bar{\phi}$. Finally, note that $\alpha^{\epsilon} \rightarrow \bar{\alpha}$ in $L^{p}$, while $\xi^{\epsilon} \star H^{*}(\cdot, \psi) \rightarrow$ $H^{*}(\cdot, \psi)$ uniformly. Letting $\epsilon \rightarrow 0+$, we are left with

$$
\begin{aligned}
& -\int_{\mathbb{T}^{d}} \zeta(T) \phi_{T}+\int_{0}^{T} \int_{\mathbb{T}^{d}} \bar{\phi} \partial_{t} \zeta+\langle D \zeta, A D \bar{\phi}\rangle+\zeta\left(\partial_{i} A_{i j} \partial_{j} \bar{\phi}+\psi \cdot D \bar{\phi}\right) \\
& \quad \leq \int_{0}^{T} \int_{\mathbb{T}^{d}} \zeta\left(\bar{\alpha}+H^{*}(\cdot, \psi)\right) .
\end{aligned}
$$

Now since $\psi$ is an arbitrary smooth vector field, we may take a sequence that approximates $\partial_{p} H(x, D \bar{\phi})$ in $L^{r^{\prime}}$. By the convexity of $H(x, \cdot)$ this yields $(40)$, as desired. 


\subsection{Stability}

We now consider the stability of solutions with respect to the data: assume that $\left(A^{n}\right),\left(H^{n}\right),\left(f^{n}\right),\left(m_{0}^{n}\right)$ and $\left(\phi_{T}^{n}\right)$ converge to $A, H, f, m_{0}$ and $\phi_{T}$ respectively. We investigate the convergence of the corresponding solution $\left(\phi^{n}, m^{n}\right)$ to the solution $(\phi, m)$. For this we assume that the $A^{n}, H^{n}, f^{n}, m_{0}^{n}$ and $\phi_{T}^{n}$ satisfy conditions (H1)...(H4) uniformly with respect to $n$. More precisely we suppose the following:

$\left(\mathrm{H} 1^{\prime}\right)$ The $f_{n}: \mathbb{T}^{d} \times[0,+\infty) \rightarrow \mathbb{R}$ are continuous in both variables, increasing with respect to the second variable $m$, and there exist $q>1$ and $C_{1}$ such that

$\frac{1}{C_{1}}|m|^{q-1}-C_{1} \leq f^{n}(x, m) \leq C_{1}|m|^{q-1}+C_{1} \quad \forall m \geq 0, \forall n \in \mathbb{N}$.

and

$$
f^{n}(x, 0)=0 \quad \forall x \in \mathbb{T}^{d}, \forall n \in \mathbb{N} .
$$

Moreover we suppose that $\left(f^{n}\right)$ converges locally uniformly to $f$ which satisfies $(\mathrm{H} 1)$.

(H2') The Hamiltonians $H^{n}: \mathbb{T}^{d} \times \mathbb{R}^{d} \rightarrow \mathbb{R}$ are continuous in both variables, convex and differentiable in the second variable, with $D_{p} H^{n}$ continuous in both variables, and have a superlinear growth in the gradient variable: there exist $r>1$ and $C_{2}>0$ such that

$$
\frac{1}{r C_{2}}|\xi|^{r}-C_{2} \leq H^{n}(x, \xi) \leq \frac{C_{2}}{r}|\xi|^{r}+C_{2} \quad \forall(x, \xi) \in \mathbb{T}^{d} \times \mathbb{R}^{d}, \forall n \in \mathbb{N} .
$$

We also suppose that the $\left(H^{n}\right)$ converge locally uniformly to $H$ which satisfies condition (H2).

(H3') There exists a constant $C_{3}>0$ and continuous maps $\Sigma^{n}: \mathbb{T}^{d} \rightarrow \mathbb{R}^{d \times D}$ such that $\Sigma^{n}\left(\Sigma^{n}\right)^{T}=A^{n}$ and

$$
\left|\Sigma^{n}(x)-\Sigma^{n}(y)\right| \leq C_{3}|x-y| \quad \forall x, y \in \mathbb{T}^{d}, \forall n \in \mathbb{N}
$$

with

either $r \geq p \quad$ or $\quad$ the $A^{n}$ are constant in space-time for all $n \in \mathbb{N}$.

We suppose that the $\left(A^{n}\right)$ converge locally uniformly to $A$.

$\left(\mathrm{H} 4^{\prime}\right)$ The $\phi_{T}^{n}: \mathbb{T}^{d} \rightarrow \mathbb{R}$ converge to $\phi$ in $\mathcal{C}^{2}\left(\mathbb{T}^{d}\right)$, while the $m_{0}^{n}: \mathbb{T}^{d} \rightarrow \mathbb{R}$ converge to $m_{0}$ in $C^{1}$ and are uniformly bounded below: there exists a constant $C_{4}>0$ such that $m_{0}^{n} \geq C_{4}$ for all $n$.

Note that the limit $A, H, f, m_{0}$ and $\phi_{T}$ satisfies assumptions (H1)...(H4).

Theorem 6.5. Let $\left(\phi^{n}, m^{n}\right)$ be a weak solution of (1) associated with $A^{n}, H^{n}$, $f^{n}$ and with the initial and terminal conditions $m_{0}^{n}$ and $\phi_{T}^{n}$. Assume also that the sequence $\left(\phi^{n}\right)$ is uniformly bounded below. Then $\left(m^{n}\right)$ converges strongly to $\bar{m}$ in $L^{q}$ while $\phi^{n}$ converges weakly and up to a subsequence to a map $\bar{\phi}$ in $L^{\gamma}$, where the pair $(\bar{\phi}, \bar{m})$ is a weak solution to (1).

Note that the existence of a solution $\left(\phi^{n}, m^{n}\right)$, such that $\phi^{n}$ is bounded by below, is ensured by Theorem 6.2. 
The result is a simple consequence of Theorem 6.4 and of the $\Gamma$ convergence of the corresponding variational problems.

Proof. Let us set $\left.w^{n}=-m^{n} D_{p} H^{n}\left(\cdot, D \phi^{n}\right)\right)$ and $\alpha^{n}=f\left(\cdot, m^{n}\right)$. Let $\mathcal{K}^{n}$ be the set of pairs $(\phi, \alpha) \in L^{\gamma}\left((0, T) \times \mathbb{T}^{d}\right) \times L^{p}\left((0, T) \times \mathbb{T}^{d}\right)$ such that $D \phi \in$ $L^{r}\left((0, T) \times \mathbb{T}^{d}\right)$ and which satisfy in the sense of distributions

$$
-\partial_{t} \phi-A_{i j}^{n}(x) \partial_{i j} \phi+H^{n}(x, D \phi) \leq \alpha, \quad \phi(T, \cdot) \leq \phi_{T}^{n} .
$$

We consider the functionals $\mathcal{A}^{n}$ and $\mathcal{B}^{n}$ defined on $\mathcal{K}^{n}$ and $\mathcal{K}_{1}$ respectively by

$$
\mathcal{A}^{n}(\phi, \alpha)=\int_{0}^{T} \int_{\mathbb{T}^{d}}\left(F^{n}\right)^{*}(x, \alpha(t, x)) d x d t-\int_{\mathbb{T}^{d}} \phi(0, x) m_{0}^{n}(x) d x
$$

and

$$
\begin{aligned}
\mathcal{B}^{n}(m, w)= & \int_{0}^{T} \int_{\mathbb{T}^{d}} m(t, x)\left(H^{n}\right)^{*}\left(x,-\frac{w(t, x)}{m(t, x)}\right)+F^{n}(x, m(t, x)) d x d t \\
& +\int_{\mathbb{T}^{d}} \phi_{T}^{n}(x) m(T, x) d x
\end{aligned}
$$

where $\left(H^{n}\right)^{*}, F^{n}$ and $\left(F^{n}\right)^{*}$ are defined from $H^{n}$ and $f^{n}$ as usual. According to the second part of Theorem 6.4 , the pair $\left(m^{n}, w^{n}\right)$ is a minimizer of problem (22) associated with $\mathcal{B}^{n}$, while the pair $\left(\phi^{n}, \alpha^{n}\right)$ is a minimizer of problem $(24)$ associated with $\mathcal{A}^{n}$.

We claim that

$$
\limsup _{n \rightarrow+\infty} \inf _{\mathcal{K}^{n}} \mathcal{A}^{n} \leq \inf _{\mathcal{K}} \mathcal{A} \quad \text { and } \quad \limsup _{n \rightarrow+\infty} \inf _{\mathcal{K}_{1}} \mathcal{B}^{n} \leq \inf _{\mathcal{K}_{1}} \mathcal{B}
$$

Let us explain the proof for $\mathcal{A}^{n}$, the proof for $\mathcal{B}^{n}$ being similar. For this we consider the class $\mathcal{K}_{2}^{n}$ of $C^{2}$ maps such that $\psi(T, \cdot) \leq \phi_{T}^{n}$. Following Proposition 5.2 , we have

$$
\begin{aligned}
& \inf _{\psi \in \mathcal{K}_{2}^{n}} \int_{0}^{T} \int_{\mathbb{T}^{d}}\left(F^{n}\right)^{*}\left(x,-\partial_{t} \psi-A_{i j}^{n}(x) \partial_{i j} \psi+H^{n}(x, D \psi)\right) d x d t \\
& \quad-\int_{\mathbb{T}^{d}} \psi(0) m_{0}^{n} d x=\inf _{\mathcal{K}^{n}} \mathcal{A}^{n} .
\end{aligned}
$$

Let $\psi \in C^{2}$ with $\psi(T, \cdot)=\phi_{T}$. As the map $\psi^{n}:=\psi-\left\|\phi_{T}^{n}-\phi_{T}\right\|_{\infty}$ belongs to $\mathcal{K}_{2}^{n}$, we have

$$
\begin{aligned}
\limsup _{n \rightarrow+\infty} \operatorname{in}_{\mathcal{K}^{n}} \mathcal{A}^{n} \leq & \limsup _{n \rightarrow+\infty} \int_{0}^{T} \int_{\mathbb{T}^{d}}\left(F^{n}\right)^{*}\left(x,-\partial_{t} \psi^{n}-A_{i j}^{n} \partial_{i j} \psi^{n}\right. \\
& \left.+H^{n}\left(x, D \psi^{n}\right)\right) d x d t-\int_{\mathbb{T}^{d}} \psi^{n}(0) m_{0}^{n} d x \\
= & \limsup _{n \rightarrow+\infty} \int_{0}^{T} \int_{\mathbb{T}^{d}}\left(F^{n}\right)^{*}\left(x,-\partial_{t} \psi-A_{i j}^{n} \partial_{i j} \psi\right. \\
& \left.+H^{n}(x, D \psi)\right) d x d t-\int_{\mathbb{T}^{d}} \psi(0) m_{0}^{n} d x+\left\|\phi_{T}^{n}-\phi_{T}\right\|_{\infty}
\end{aligned}
$$


Vol. 22 (2015)

$$
\begin{aligned}
\leq & \int_{0}^{T} \int_{\mathbb{T}^{d}} F^{*}\left(x,-\partial_{t} \psi-A_{i j} \partial_{i j} \psi+H(x, D \psi)\right) d x d t \\
& -\int_{\mathbb{T}^{d}} \psi(0) m_{0} d x .
\end{aligned}
$$

This proves claim (51).

Combining (51) with Lemma 4.2 and Proposition 5.2, we have

$$
\begin{aligned}
\limsup _{n \rightarrow+\infty} \inf _{\mathcal{K}^{n}} \mathcal{A}^{n} \leq \inf _{\mathcal{K}} \mathcal{A} & =-\inf _{\mathcal{K}_{1}} \mathcal{B} \leq-\limsup _{n \rightarrow+\infty} \inf _{\mathcal{K}_{1}} \mathcal{B}^{n} \\
& =\liminf _{n \rightarrow+\infty}\left(-\inf _{\mathcal{K}_{1}} \mathcal{B}^{n}\right) \leq \liminf _{n \rightarrow+\infty} \inf _{\mathcal{K}^{n}} \mathcal{A}^{n} .
\end{aligned}
$$

Since the left-hand side is not larger then the right-hand side, the above inequalities are in fact equalities, which shows that

$$
\inf _{\mathcal{K}} \mathcal{A}=-\inf _{\mathcal{K}_{1}} \mathcal{B}=\lim _{n \rightarrow+\infty} \inf _{\mathcal{K}^{n}} \mathcal{A}^{n}=-\lim _{n \rightarrow+\infty} \inf _{\mathcal{K}_{1}} \mathcal{B}^{n}
$$

We now show that the sequence $\left(m^{n}, w^{n}\right)$ converges to a minimizer of $\mathcal{B}$. Using the estimates established for the proof of Proposition 5.2, we have

$$
\left\|m^{n}\right\|_{L^{q}}+\left\|w^{n}\right\|_{L^{\frac{r^{\prime} q}{r^{\prime}+q-1}}}+\left\|-w^{n} / m^{n}\right\|_{L_{m^{n}}^{r^{\prime}}} \leq C
$$

By Lemma 4.1, this implies that the maps $t \rightarrow m^{n}(t)$ are uniformly Hölder continuous in $P\left(\mathbb{T}^{d}\right)$. Hence there is a subsequence of $\left(m^{n}, w^{n}\right)$ (still denoted in the same way) which converges weakly in $L^{q} \times L^{\frac{r^{\prime} q}{r^{\prime}+q-1}}$ to some $(\bar{m}, \bar{w}) \in \mathcal{K}_{1}$ and which is such that $\left(m^{n}(t)\right)$ converges to $(\bar{m}(t))$ in $C^{0}\left([0, T], P\left(\mathbb{T}^{d}\right)\right)$. Then, for any $\xi \in L^{\infty}\left((0, T) \times \mathbb{T}^{d}, \mathbb{R}^{d}\right)$ and $\sigma \in L^{\infty}\left((0, T) \times \mathbb{T}^{d}\right)$ we have

$$
\begin{aligned}
\liminf _{n \rightarrow+\infty} \mathcal{B}^{n}\left(m^{n}, w^{n}\right) \geq & \liminf _{n \rightarrow+\infty} \int_{0}^{T} \int_{\mathbb{T}^{d}}\left\langle\xi, w^{n}\right\rangle-H(x, \xi) \\
& +\sigma m^{n}-F^{*}(x, \sigma) d x d t+\int_{\mathbb{T}^{d}} m^{n}(T) \phi_{T}^{n} d x \\
\geq & \int_{0}^{T} \int_{\mathbb{T}^{d}}\langle\xi, \bar{w}\rangle-H(x, \xi)+\sigma \bar{m}-F^{*}(x, \sigma) d x d t \\
& +\int_{\mathbb{T}^{d}} \bar{m}(T) \phi_{T} d x
\end{aligned}
$$

Taking the supremum with respect to $(\xi, \sigma)$ and using $(52)$ then gives

$$
\inf _{\mathcal{K}_{1}} \mathcal{B}=\lim _{n \rightarrow \infty} \inf _{\mathcal{K}_{1}} \mathcal{B}^{n}=\liminf _{n \rightarrow+\infty} \mathcal{B}^{n}\left(m^{n}, w^{n}\right) \geq \mathcal{B}(\bar{m}, \bar{w})
$$

Hence $(\bar{m}, \bar{w})$ minimizes $\mathcal{B}$. Moreover we note for later use that the above equalities entail that 


$$
\int_{0}^{T} \int_{\mathbb{T}^{d}} F(x, m) d x d t=\lim _{n \rightarrow+\infty} \int_{0}^{T} \int_{\mathbb{T}^{d}} F^{n}\left(x, m^{n}\right) d x d t .
$$

In order to prove the strong convergence of $\left(m^{n}, w^{n}\right)$, we rely on a standard argument using Young measures. Let us define the sequence of measures $\left(\mu^{n}\right)$ on $[0, T] \times \mathbb{T}^{d} \times \mathbb{R}$ by $d \mu^{n}(t, x, \rho)=d t d x d \delta_{m^{n}(t, x)}(\rho)$. In view of (53), the sequence $\left(\mu^{n}\right)$ is tight and therefore converges weakly to some measure $\mu$ which can be disintegrated into $d \mu(t, x, \rho)=d \mu_{t, x}(\rho) d t d x$. Then following Theorem 6.11 of [22], we have, as the $F^{n}$ are bounded below:

$$
\begin{aligned}
\int_{0}^{T} \int_{\mathbb{T}^{d}} F(x, m) d x d t & =\lim _{n \rightarrow+\infty} \int_{0}^{T} \int_{\mathbb{T}^{d}} F^{n}\left(x, m^{n}\right) d x d t \\
& \geq \int_{0}^{T} \int_{\mathbb{T}^{d}} \int_{\mathbb{R}} F(x, \rho) d \mu_{t, x}(\rho) d x d t,
\end{aligned}
$$

where, by convexity of $F$,

$$
\begin{aligned}
\int_{0}^{T} \int_{\mathbb{T}^{d}} \int_{\mathbb{R}} F(x, \rho) d \mu_{t, x}(\rho) d x d t & \geq \int_{0}^{T} \int_{\mathbb{T}^{d}} F\left(x, \int_{\mathbb{R}} \rho d \mu_{t, x}(\rho)\right) d x d t \\
& =\int_{0}^{T} \int_{\mathbb{T}^{d}} F(x, m) d x d t .
\end{aligned}
$$

By strict convexity of $F$, this implies that $\mu_{t, x}=\delta_{m(t, x)}$ a.e., which means that the sequence $\left(m^{n}\right)$ actually strongly converges to $m$ in $L^{q}$ (Proposition 6.12 of [22]). The strong convergence of the sequence $\left(w^{n}\right)$ can be checked in the same way, by using the strict convexity of $H^{*}$. So we have checked that any converging subsequence of $\left(m^{n}, w^{n}\right)$ strongly converges to a minimizer $(\bar{m}, \bar{w})$ of $\mathcal{B}$ on $\mathcal{K}_{1}$. Since this minimizer is unique, the full sequence $\left(m^{n}, w^{n}\right)$ strongly converges to $(\bar{m}, \bar{w})$.

Next we turn to the convergence of $\left(\phi^{n}, \alpha^{n}\right)$. The growth condition (2) on $f$ implies that the sequence $\left(\alpha^{n}=f^{n}\left(\cdot, m^{n}\right)\right)$ converges in $L^{p}$ to $\bar{\alpha}:=f(\cdot, \bar{m})$. As $\left(\phi^{n}\right)$ is uniformly bounded below, Theorem 3.3 implies that

$$
\left\|\phi^{n}\right\|_{L^{\infty}\left((0, T), L^{\eta}\left(\mathbb{T}^{d}\right)\right)}+\left\|\phi^{n}\right\|_{L^{\gamma}\left((0, T) \times \mathbb{T}^{d}\right)} \leq C .
$$

The end of the proof follows closely the argument in Proposition 5.4: $\left(D \phi^{n}\right)$ is bounded in $L^{r}$, so that, up to a subsequence, $\left(\phi^{n}\right)$ converges weakly to some $\bar{\phi}$ in $L^{\gamma}$ while $D \phi^{n}$ converges weakly to $D \bar{\phi}$ in $L^{r}$. Using the same argument as in Proposition 5.4, one can check that $(\bar{\phi}, \bar{\alpha})$ belongs to $\mathcal{K}$ and is a minimizer of $\mathcal{A}$ over $\mathcal{K}$. Theorem 6.4 then states that the pair $(\bar{\phi}, \bar{m})$ is a solution to the MFG problem (1).

\section{Acknowledgements}

We wish to thank the anonymous referee for useful comments. This work has been partially supported by the Commission of the European Communities under the 7th Framework Programme Marie Curie Initial Training Networks Project SADCO, FP7-PEOPLE-2010-ITN, No 264735, by the French National Research Agency ANR-10-BLAN 0112 and ANR-12-BS01-0008-01 and by the 
Italian Indam Gnampa project 2013 "Modelli di campo medio nelle dinamiche di popolazioni e giochi differenziali".

\section{References}

[1] Benamou, J.D., Brenier, Y.: A computational fluid mechanics solution to the Monge-Kantorovich mass transfer problem. Numer. Math. 84, 375-393 (2000)

[2] Carmona, R., Delarue, F. Probabilist analysis of Mean-Field Games. SIAM J. Control Optim. 51(4), 2705-2734 (2013)

[3] Cardaliaguet, P.: Weak solutions for first order mean field games with local coupling. To appear in Springer-INdAM volume "Analysis and Geometry in Control Theory and its Applications" (2015)

[4] Cardaliaguet, P., Graber, P.J.: Mean field games systems of first order. To appear in ESAIM COCV (2015)

[5] Cardaliaguet P., Carlier G., Nazaret B.: Geodesics for a class of distances in the space of probability measures. Calc. Var. Partial Differ. Equ. 1-26 (2012)

[6] Cardaliaguet, P., Silvestre, L.: Hölder continuity to Hamilton-Jacobi equations with superquadratic growth in the gradient and unbounded right-hand side. Commun. Partial Differ. Eq. 37(9), 1668-1688 (2012)

[7] Cardaliaguet, P., Lasry, J.-M., Lions, P.-L., Porretta, A.: Long time average of mean field games. Netw. Heterog. Media 7(2):279-301 (2012)

[8] DiBenedetto E.: Degenerate parabolic equations. Springer (1993)

[9] DiPerna, R., Lions, P.-L.: Ordinary differential equations, transport theory and Sobolev spaces. Invent. Math. 98(3), 511-547 (1989)

[10] Ekeland, I., Temam, R.: Convex analysis and variational problems, english ed., vol. 28 of Classics in Applied Mathematics. Society for Industrial and Applied Mathematics (SIAM), Philadelphia, PA, (1999) (Translated from the French)

[11] Gomes, D. A., Pimentel, E.: Time dependent mean-field games with logarithmic nonlinearities. arXiv preprint arXiv:1407.1261 (2014)

[12] Gomes, D. A., Pimentel, E., Sánchez-Morgado, H.: Time dependent meanfield games in the subquadratic case. To appear in Communications in Partial Differential Equations arXiv preprint arXiv:1311.6684 (2013)

[13] Gomes, D. A., Pimentel, E., Sánchez-Morgado, H.: Time dependent mean-field games in the superquadratic case. arXiv preprint arXiv:1311.6684 (2013)

[14] Graber P.J.: Optimal control of first-order Hamilton-Jacobi equations with linearly bounded Hamiltonian, Appl. Math. Optim. 70(2), 185-224 (2014)

[15] Huang, M., Malhamé, R.P., Caines, P.E.: Large population stochastic dynamic games: closed-loop McKean-Vlasov systems and the Nash certainty equivalence principle. Commun. Inf. Syst. 6(3), 221-252 (2006) 
[16] Ishii, H.: On the equivalence of two notions of weak solutions, viscosity solutions and distribution solutions. Funkcial. Ekvac 38(1), 101-120 (1995)

[17] Lasry, J.-M., Lions, P.-L.: Jeux à champ moyen. I. Le cas stationnaire. C. R. Math. Acad. Sci. Paris 343(9), 619-625 (2006)

[18] Lasry, J.-M., Lions, P.-L.: Jeux à champ moyen. II. Horizon fini et contrôle optimal. C. R. Math. Acad. Sci. Paris 343(10), 679-684 (2006)

[19] Lasry, J.-M., Lions, P.-L.: Mean field games. Jpn. J. Math. 2(1), 229-260 (2007)

[20] Le Bris, C., Lions, P.-L.: Existence and uniqueness of solutions to FokkerPlanck type equations with irregular coefficients. Commun. Partial Differ. Equ. 33(7), 1272-1317 (2008)

[21] Lions, P.L.: In Cours au Collège de France. http://www.college-de-france.fr

[22] Pedregal, P.: Parametrized measures and variational principles, vol. 30. Springer Science \& Business Media (1997)

[23] Porretta, A.: Weak solutions to Fokker-Planck equations and mean field games. Arch. Ration. Mech. Anal. 216(1), 1-62 (2015)

[24] Stampacchia, G.: Le problème de Dirichlet pour les équations elliptiques du seconde ordre à coefficients discontinus. Ann. Inst. Fourier (Grenoble) 15, 189258 (1965)

Pierre Cardaliaguet and Daniela Tonon

Ceremade, Université Paris-Dauphine

Place du Maréchal de Lattre de Tassigny

75775 Paris cedex 16

France

e-mail: cardaliaguet@ceremade.dauphine.fr

Daniela Tonon

e-mail: tonon@ceremade.dauphine.fr

P. Jameson Graber

828, Boulevard des Maréchaux

91762 Palaiseau Cedex

France

e-mail: jameson.graber@ensta-paristech.fr 


\author{
Alessio Porretta \\ Dipartimento di Matematica \\ Università di Roma "Tor Vergata" \\ Via della Ricerca Scientifica 1 \\ 00133 Roma \\ Italy \\ e-mail:porretta@mat.uniroma2.it
}

Received: 25 July 2014.

Accepted: 18 March 2015. 